\title{
Harmonic analysis of isotropic fields on the sphere with arbitrary masks
}

\author{
Henry S. Grasshorn Gebhardt ${ }^{a, b}$ and Olivier Doréa ${ }^{a, b}$ \\ ${ }^{a}$ Jet Propulsion Laboratory, California Institute of Technology, Pasadena, CA 91109, USA \\ ${ }^{b}$ California Institute of Technology, Pasadena, CA 91125, USA \\ E-mail: henry.s.gebhardt@jpl.nasa.gov, olivier.p.dore@jpl.nasa.gov
}

\begin{abstract}
Obtaining constraints from the largest scales of a galaxy survey is challenging due to the survey mask allowing only partial measurement of large angular modes. This scatters information from the harmonic-space 2-point function away from the diagonal and introduces coupling between modes. In this paper, we derive a custom eigenbasis adapted to any particular survey geometry so that all information is retained on the diagonal. At the expense of a somewhat complex pixel- and selection-function-window, the result is a diagonal 2-point function with a simple shot noise, and a diagonal covariance matrix in the case of a Gaussian random field. We derive the basis on the surface of a sphere, and we use it to construct a 3D spherical Fourier-Bessel power spectrum estimator assuming a survey geometry that is separable in the angular and radial directions.
\end{abstract}




\section{Contents}

1 Introduction 1

2 Laplaceology 3

3 Angular Eigenfunctions $\quad 4$

3.1 Integral operator 4

3.2 Green's Function 6

3.3 The Monopole 6

3.4 The Cryobasis 6

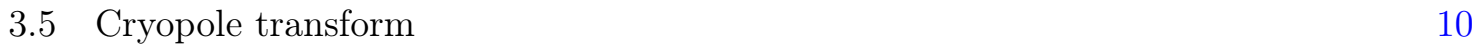

$\begin{array}{lll}3.6 & \text { Cryopower } & 11\end{array}$

3.7 The cryo-window 12

$\begin{array}{lll}3.7 .1 & \text { Continuous bases } & 13\end{array}$

$\begin{array}{lll}3.8 & \text { Shot noise } & 15\end{array}$

4 Spherical Fourier-Bessel decomposition $\quad 16$

$\begin{array}{lll}4.1 & \text { Radial Modes } & 17\end{array}$

$\begin{array}{ll}4.2 \text { Into Cryospace } & 20\end{array}$

$\begin{array}{ll}4.3 \text { Cryo-Window } & 20\end{array}$

4.4 Local Average Effect 22

4.5 SFB Cryopower 24

4.6 Covariance matrix 24

5 Discussion 28

5.1 Non-Local Boundary Conditions 28

$\begin{array}{lll}5.2 & \text { Interpretation of effective } \ell \text { and } m & 29\end{array}$

$\begin{array}{lll}5.3 & \text { Relation to [1] } & 29\end{array}$

6 Conclusion 31

A Useful formulae $\quad 33$

B Radial Green's function $\quad 33$

B.1 First derivation 34

B.2 Another derivation 34

\section{Introduction}

For galaxy surveys over the full sky, spherical harmonics $Y_{\ell m}(\hat{\boldsymbol{r}})$ provide a convenient basis for the analysis of an isotropic field that accounts for all wide-angle effects. In practice, however, galaxy surveys are restricted to the partial sky, e.g., due to the exclusion zone of the Milky Way or due to time constraints of a deep survey. This non-isotropic mask scatters information to off-diagonal terms in the harmonic/Fourier-space 2-point function, which typically leads to a pseudo- $C_{\ell}$ power spectrum with coupled modes and a complicated covariance matrix. 
For a homogeneous and isotropic random field in 3D or 2D, the 2-point function, formally constructed as the outer product of the field, becomes diagonal in the eigenbasis of the Laplacian when restricted to the domain of the survey (see Section 2 for details). The basis functions must satisfy

$$
\nabla^{2} f=-k^{2} f
$$

anywhere within the survey. For example, if the survey is a rectangular box in 3D, then the eigenfunctions are of the form $e^{i \boldsymbol{k} \cdot \boldsymbol{x}}$. On the 2D sphere, the eigenfunctions are the spherical harmonics $Y_{\ell m}(\hat{\boldsymbol{r}})$, and adding the radial direction for the full volume of a sphere results in the spherical Fourier-Bessel (SFB) basis $j_{\ell}(k r) Y_{\ell m}(\hat{\boldsymbol{r}})$, where $j_{\ell}(k r)$ are spherical Bessel functions. The SFB basis is well-suited for the radial/angular separation, and in [2] we allow the survey geometry to be a thick spherical shell, in which case the spherical Bessels become superpositions of spherical Bessels of the first and second kind [3].

In this paper, we push this concept further by deriving an eigenbasis of the Laplacian for arbitrary masks. For example, in a typical Fourier-analysis with a rectangular analysis box, parts of the box are left empty, and this leads to the scattering of information into offdiagonal terms and coupling between modes. By adapting the basis functions to the survey geometry, we essentially fit the Fourier-analysis box perfectly onto the survey. However, as a simplification we assume that the radial selection function and angular masks are separable.

Several convenient properties follow from our procedure. First, no information is scattered to off-diagonal terms in the 2-point function and modes are not coupled by the window function. Second, Poissonian shot noise is simply $1 / \bar{n}$ and the local average effect (or integral constraint) can be modeled in a very simple fashion, eliminating power in the $\ell=0$ modes, only. Third, to leading order the covariance matrix is diagonal and no harder to calculate than modeling the 2-point function. Thus, compared to traditional methods, we avoid the need for pseudo- $C_{\ell}$ and complicated coupling matrices, and we have a significantly reduced computational cost for producing an analytical covariance matrix.

Our method has two main downsides. First, the survey geometry and pixel window both enter the observed power spectrum in a nontrivial way, and this leads to a somewhat complicated combination of pixel and geometric window that cannot be easily inverted. Second, with an arbitrary mask, Eq. (1.1) does not have an analytical solution and must be solved numerically. Therefore, our method is currently limited to only large scales due to the high up-front computational cost of deriving the eigenfunctions, which typically scales as $N^{3}$, where $N$ is the number of pixels on the sky or radial bins (as we assume separable radial and angular masks). In our current implementation, with a modern laptop, this allows the number of pixels $N$ to be up to about $10^{4}$ or $10^{5}$, corresponding to a resolution $n_{\text {side }}=32$ or 64 for a full-sky survey, and correspondingly higher resolution for a smaller survey area.

In this paper, we follow $[4,5]$ to convert Eq. (1.1) into an integral equation that is readily adapted to arbitrary survey geometries, including disconnected geometries, e.g., when there are northern and southern survey areas. We will differ from [5] by using the HEALPix [6] scheme for pixelization on a sphere. We show that the resulting eigenfunctions are linear combinations of spherical harmonics with effective non-integer $\ell$-modes.

The method relies on the calculation of eigenfunctions to the Laplacian, adapted to a specific survey geometry. That is, we let the reader create your own funktions (CRYOFUNK).

We assume that the radial selection and angular mask are separable, for two reasons. First, since the SFB power spectrum is dependent on both $\ell$ and $k$ modes, we wish to retain the ability to assign a definitive $\ell$ to each mode. Second, if we were to combine them, the 
number of voxels may be prohibitively large so that the method could only be used for an extremely small number of modes.

We start in Section 2 by discussing the use of the Laplacian as a generator for the basis functions. In Section 3 we review in detail the essential parts of [5] for deriving angular basis functions, and we extend their results to general pixelization schemes, focussing on HEALPix. We also derive the combined pixel- and selection-window and the shot noise. In Section 4 we then apply the method to the radial basis functions in order to construct a full SFB power spectrum estimator. In Section 5 we add some discussion points, and we conclude in Section 6. Appendix A contains some useful formulae, and in Appendix B we derive the radial Green's function.

Our code will be available publically at https://github.com/hsgg/CryoFaBs.jl, once approved for release by our institution.

\section{Laplaceology}

In this section we aim to explain the use of the Laplacian as a generator for eigenfunctions that will be useful for cosmological analysis. In effect, the harmonic transform generated by the Laplacian diagonalizes the 2-point function of a homogeneous field, which then represents an efficient compression of the data with a simple covariance matrix.

The symmetries to exploit are the translational and rotational invariance of the statistical field. Rotational invariance manifests itself as a 2D-translational invariance on the curved sky, so here we will only consider translational invariance explicitly. Translational invariance in the radial direction is broken by line-of-sight effects such as the growth of structure. However, as long as such effects are statistically slowly varying with redshift, assumption of invariance will still give a convenient basis.

To demand compression is to demand that the power spectrum is the diagonal form of the correlation function. To make this more precise, we define

$$
\begin{aligned}
\xi\left(\boldsymbol{r}, \boldsymbol{r}^{\prime}\right) & =\left\langle\delta(\boldsymbol{r}) \delta\left(\boldsymbol{r}^{\prime}\right)\right\rangle, \\
P\left(\boldsymbol{k}, \boldsymbol{k}^{\prime}\right) & =\left\langle\delta(\boldsymbol{k}) \delta^{*}\left(\boldsymbol{k}^{\prime}\right)\right\rangle .
\end{aligned}
$$

If our basis functions are $f(\boldsymbol{k}, \boldsymbol{r})$, then these second moments are related by

$$
P\left(\boldsymbol{k}, \boldsymbol{k}^{\prime}\right)=\int \mathrm{d}^{3} r W(\boldsymbol{r}) f(\boldsymbol{k}, \boldsymbol{r}) \int \mathrm{d}^{3} r^{\prime} W\left(\boldsymbol{r}^{\prime}\right) f^{*}\left(\boldsymbol{k}^{\prime}, \boldsymbol{r}^{\prime}\right) \xi\left(\boldsymbol{r}, \boldsymbol{r}^{\prime}\right),
$$

where the window function $W(\boldsymbol{r})=1$ inside the survey and vanishes outside it.

To exploit the translational invariance, we assume that there is some transformation parameterized by $\lambda^{\mu}$ that leaves the correlation function $\xi\left(\boldsymbol{r}, \boldsymbol{r}^{\prime}\right)$ invariant. In our case $\lambda^{\mu}$ parameterizes a translation. Then, the correlation function is $\xi\left(\boldsymbol{r}+\lambda^{\mu}, \boldsymbol{r}^{\prime}+\lambda^{\mu}\right)$ with $\mu=1,2,3$, and homogeneity demands that the first derivative of $\xi$ w.r.t. any parameter $\lambda^{\mu}$ vanishes. Further, we can choose $\lambda^{\mu}=-\boldsymbol{r}$ to show that the correlation function only depends on $s=\boldsymbol{r}^{\prime}-\boldsymbol{r}$ under this symmetry.

With this translation symmetry, Eq. (2.3) becomes

$$
P\left(\boldsymbol{k}, \boldsymbol{k}^{\prime}\right)=\int \mathrm{d}^{3} s \int \mathrm{d}^{3} r W(\boldsymbol{r}) f(\boldsymbol{k}, \boldsymbol{r}) W(\boldsymbol{r}+\boldsymbol{s}) f^{*}\left(\boldsymbol{k}^{\prime}, \boldsymbol{r}+\boldsymbol{s}\right) \xi(\boldsymbol{s}) .
$$


Diagonalization is achieved when the basis functions $f(\boldsymbol{k}, \boldsymbol{r})$ satisfy

$$
\int \mathrm{d}^{3} r[W(\boldsymbol{r}) f(\boldsymbol{k}, \boldsymbol{r})]\left[W(\boldsymbol{r}+\boldsymbol{s}) f^{*}\left(\boldsymbol{k}^{\prime}, \boldsymbol{r}+\boldsymbol{s}\right)\right]=\delta^{D}\left(\boldsymbol{k}^{\prime}-\boldsymbol{k}\right) g(\boldsymbol{k}, \boldsymbol{s}),
$$

for some function $g(\boldsymbol{k}, s)$. For example, if $W(\boldsymbol{r})=1$ everywhere in $\mathbb{R}^{3}$, then the standard Fourier basis $f(\boldsymbol{k}, \boldsymbol{r})=e^{-i \boldsymbol{k} \cdot \boldsymbol{r}}$ satisfies this relation with $g(\boldsymbol{k}, \boldsymbol{s})=(2 \pi)^{3} e^{i \boldsymbol{k} \cdot \boldsymbol{s}}$.

For compression, Eq. (2.5) must be satisfied for all $\boldsymbol{s}$. This is especially true for an infinitesimally small $s$. In the limit $s \rightarrow 0$, Eq. (2.5) becomes the orthogonality condition

$$
\int \mathrm{d}^{3} r[W(\boldsymbol{r}) f(\boldsymbol{k}, \boldsymbol{r})]\left[W(\boldsymbol{r}) f^{*}\left(\boldsymbol{k}^{\prime}, \boldsymbol{r}\right)\right]=\delta^{D}\left(\boldsymbol{k}^{\prime}-\boldsymbol{k}\right) g(\boldsymbol{k}, 0) .
$$

Application of the gradient w.r.t. $s$ in Eq. (2.5), and taking the limit $s \rightarrow 0$ gives the further condition

$$
\int \mathrm{d}^{3} r[W(\boldsymbol{r}) f(\boldsymbol{k}, \boldsymbol{r})] \nabla_{\boldsymbol{r}}\left[W(\boldsymbol{r}) f^{*}\left(\boldsymbol{k}^{\prime}, \boldsymbol{r}\right)\right]=\left.\delta^{D}\left(\boldsymbol{k}^{\prime}-\boldsymbol{k}\right) \nabla_{\boldsymbol{s}} g(\boldsymbol{k}, \boldsymbol{s})\right|_{s=0} .
$$

Thus, the demand is that the basis functions $f(\boldsymbol{k}, \boldsymbol{r})$ are orthogonal functions over the domain of the survey, and they are also orthogonal to their gradient provided that $\boldsymbol{k} \neq \boldsymbol{k}^{\prime}$.

As is evident from Eq. (2.7), choosing the basis functions $f(\boldsymbol{k}, \boldsymbol{r})$ to be eigenfunctions of the gradient $\nabla_{\boldsymbol{r}}$ will satisfy Eqs. (2.6) and (2.7).

Applying the gradient twice to an eigenfunction, it is further evident that any eigenfunction of the gradient $\nabla_{\boldsymbol{r}}$ is also an eigenfunction of the Laplacian $\nabla_{\boldsymbol{r}}^{2}$. Indeed, the set of eigenfunctions to the Laplacian also satisfies Eqs. (2.6) and (2.7).

A caveat in our derivation is that, strictly speaking, we have limited our results to only infinitesimal $s$. However, repeatedly taking derivatives w.r.t. $s$ allows us to build up an infinite series of conditions. Indeed, we could have derived Eqs. (2.6) and (2.7) by expanding $W(\boldsymbol{r}+\boldsymbol{s}) f^{*}\left(\boldsymbol{k}^{\prime}, \boldsymbol{r}+\boldsymbol{s}\right)$ in a Taylor series, and the higher-order terms would lead to exactly these additional conditions. Therefore, our result that $W(\boldsymbol{r}) f(\boldsymbol{k}, \boldsymbol{r})$ as Laplacian eigenfunctions leads to a diagonal 2-point function holds for finite $s$ as well in a wide variety of cases.

Therefore, using eigenfunctions of the Laplacian gives us a basis that exploits the translational symmetry for the 2-point function. This also holds true for the isotropy in $2 \mathrm{D}$ on the spherical sky, which is essentially a translation symmetry for the sky position $\hat{\boldsymbol{r}}$. Furthermore, as we will show in Section 4.6, for a Gaussian random field this also means that the covariance matrix will have a very simple form, namely it will be diagonal.

\section{Angular Eigenfunctions}

In this section we extend [5] using the HEALPix scheme. The key idea is to convert the differential equation Eq. (1.1) to an integral equation using the Green's function, because the integral equation lends itself naturally to a generalization to disconnected domains. Furthermore, the use of the HEALPix scheme allows some simplification and better numerical accuracy.

\subsection{Integral operator}

Instead of using the differential operator $\nabla^{2},[5]$ use the equivalent integral equation, derived as follows. The Green's function $G\left(\hat{\boldsymbol{r}}, \hat{\boldsymbol{r}}^{\prime}\right)$ is the response of a signal at $\hat{\boldsymbol{r}}^{\prime}$ on the pixel at $\hat{\boldsymbol{r}}$. For the Laplacian, the Green's function is the solution to the equation

$$
\nabla^{2} G\left(\hat{\boldsymbol{r}}, \hat{\boldsymbol{r}}^{\prime}\right)=-\delta^{D}\left(\hat{\boldsymbol{r}}^{\prime}-\hat{\boldsymbol{r}}\right) .
$$


That is, the Green's function is the inverse to the Laplacian, $\nabla^{-2}$. Then, for some arbitrary source function $f(\hat{\boldsymbol{r}})$, the equation

$$
\nabla^{2} Y=-f(\hat{\boldsymbol{r}})
$$

has the solution

$$
Y(\hat{\boldsymbol{r}})=\int_{D} \mathrm{~d}^{2} \hat{\boldsymbol{r}}^{\prime} G\left(\hat{\boldsymbol{r}}, \hat{\boldsymbol{r}}^{\prime}\right) f\left(\hat{\boldsymbol{r}}^{\prime}\right)
$$

on the domain $D$. To solve the Helmholtz equation Eq. (1.1) and define the harmonic basis functions, one can substitute $f=\lambda Y$ and obtain

$$
Y(\hat{\boldsymbol{r}})=\lambda \int_{D} \mathrm{~d}^{2} \hat{\boldsymbol{r}}^{\prime} G\left(\hat{\boldsymbol{r}}, \hat{\boldsymbol{r}}^{\prime}\right) Y\left(\hat{\boldsymbol{r}}^{\prime}\right) .
$$

The Fredholm equation Eq. (3.4) is formally equivalent to Eq. (1.1). However, Eq. (3.4) is more readily adapted to arbitrary boundaries, simply by changing the integration domain.

To discretize, we integrate Eq. (3.4) over the pixel $i$, or define

$$
z_{i}=\Omega_{i}^{s} \int \mathrm{d}^{2} \hat{\boldsymbol{r}} w_{i}(\hat{\boldsymbol{r}}) Y(\hat{\boldsymbol{r}})
$$

where $\Omega_{i}$ is the area of pixel $i$, the parameter $s$ is chosen so that the operator $G_{i j}$ defined below becomes symmetric, and the window $w_{i}(\hat{\boldsymbol{r}})=1 / \Omega_{i}$ within the area of pixel $i$ and vanishes outside. The average value of $Y\left(\hat{\boldsymbol{r}}^{\prime}\right)$ over the pixel $j$ is then $\Omega_{j}^{-s} z_{j}$. Next, split the integral in Eq. (3.4) into a sum over pixels $j$, and integrate over pixel $i$,

$$
\begin{aligned}
z_{i} & =\lambda \Omega_{i}^{s} \int \mathrm{d}^{2} \hat{\boldsymbol{r}} w_{i}(\hat{\boldsymbol{r}}) \sum_{j} \Omega_{j} \int \mathrm{d}^{2} \hat{\boldsymbol{r}}^{\prime} w_{j}\left(\hat{\boldsymbol{r}}^{\prime}\right) G\left(\hat{\boldsymbol{r}}, \hat{\boldsymbol{r}}^{\prime}\right) Y\left(\hat{\boldsymbol{r}}^{\prime}\right) \\
& \approx \lambda \sum_{j} \Omega_{i}^{s} \Omega_{j}^{1-s} z_{j} \int \mathrm{d}^{2} \hat{\boldsymbol{r}} w_{i}(\hat{\boldsymbol{r}}) \int \mathrm{d}^{2} \hat{\boldsymbol{r}}^{\prime} w_{j}\left(\hat{\boldsymbol{r}}^{\prime}\right) G\left(\hat{\boldsymbol{r}}, \hat{\boldsymbol{r}}^{\prime}\right),
\end{aligned}
$$

where we make the assumption that $Y\left(\hat{\boldsymbol{r}}^{\prime}\right)$ does not vary much across the pixel $j$ and it can be pulled out of the integral. Written in matrix form, we get the eigenequation

$$
\mathbf{z} \approx \lambda \mathbf{G} \mathbf{z}
$$

where we defined the Green's matrix $\mathbf{G}$ with elements

$$
G_{i j}=\Omega_{i}^{s} \Omega_{j}^{1-s} \int \mathrm{d}^{2} \hat{\boldsymbol{r}} w_{i}(\hat{\boldsymbol{r}}) \int \mathrm{d}^{2} \hat{\boldsymbol{r}}^{\prime} w_{j}\left(\hat{\boldsymbol{r}}^{\prime}\right) G\left(\hat{\boldsymbol{r}}, \hat{\boldsymbol{r}}^{\prime}\right) .
$$

$G_{i j}$ is the response of pixel $i$ to a signal in pixel $j$. As Eq. (3.1) shows, $G$ is symmetric, i.e., $G\left(\hat{\boldsymbol{r}}, \hat{\boldsymbol{r}}^{\prime}\right)=G\left(\hat{\boldsymbol{r}}^{\prime}, \hat{\boldsymbol{r}}\right)$, and so $G_{i j}=G_{j i}$ is also symmetric, provided we either choose

$$
s=\frac{1}{2},
$$

or we choose a pixelization scheme such as HEALPix where all pixels have the same area $\Omega_{i}$.

The basis functions satisfying Eq. (3.8) are linear combinations of the spherical harmonics, as we show in Section 5. Combining $Y_{\ell m}$ with differeing $\ell$, therefore, may lead to an effective non-integer $\ell$ for individual basis functions. 


\subsection{Green's Function}

The Green's function for the Laplacian on a sphere is given by

$$
G\left(\hat{\boldsymbol{r}}, \hat{\boldsymbol{r}}^{\prime}\right)=-\frac{1}{4 \pi} \ln \left(2 \sin ^{2} \frac{\rho}{2}\right),
$$

[e.g. 4,5$]$ where the great circle distance $\rho$ between $\hat{\boldsymbol{r}}$ and $\hat{\boldsymbol{r}}^{\prime}$ is

$$
\rho=\arcsin \left|\hat{\boldsymbol{r}} \times \hat{\boldsymbol{r}}^{\prime}\right|=2 \arcsin \sqrt{\sin ^{2} \frac{\Delta \theta}{2}+\sin \theta \sin \theta^{\prime} \sin ^{2} \frac{\Delta \phi}{2}},
$$

where $\theta$ is the angle used in the HEALPix convention, and $\Delta \theta=\theta^{\prime}-\theta$ and $\Delta \phi=\phi^{\prime}-\phi$. The Haversine formula Eq. (3.12) is numerically stable for small distances, and it is sufficient for the use case in this paper. We follow [5] and use the value of the Green's function at the center of the pixel when $\rho \neq 0$. That is, inserting Eq. (3.12) into Eqs. (3.9) and (3.11), we get for $i \neq j$

$$
G_{i j} \simeq-\frac{\Omega_{i}^{s} \Omega_{j}^{1-s}}{4 \pi} \ln \left(2 \sin ^{2} \frac{\theta_{j}-\theta_{i}}{2}+2 \sin \theta_{i} \sin \theta_{j} \sin ^{2} \frac{\phi_{j}-\phi_{i}}{2}\right),
$$

where the integrals contributed a factor $\Omega_{i} \Omega_{j}$.

When $\rho=0$ Green's function Eq. (3.11) diverges, and so for $i=j$ we approximate the matrix element by integrating over a circular area the same size of a pixel. Eq. (3.9) becomes

$$
\begin{aligned}
G_{i i} & \approx \frac{1}{\Omega_{i}} \int_{i} \mathrm{~d}^{2} \hat{\boldsymbol{r}} 2 \pi \int_{0}^{\rho_{i}} \mathrm{~d} \rho \rho\left[-\frac{1}{2 \pi} \ln \left(\frac{\rho}{\sqrt{2}}\right)\right] \\
& \approx \frac{\rho_{i}^{2}}{4}\left(1-\ln \frac{\rho_{i}^{2}}{2}\right) .
\end{aligned}
$$

Since $\rho_{i}$ is the radius of a circle with the area $\Omega_{i}$, we have that $\pi \rho_{i}^{2} \simeq \Omega_{i}$, and

$$
G_{i i} \approx \frac{\Omega_{i}}{4 \pi}\left(1-\ln \frac{\Omega_{i}}{2 \pi}\right) .
$$

\subsection{The Monopole}

In transforming the Helmholtz Eq. (1.1) to a Fredholm Eq. (3.4), we ignored the monopole solution: $Y=$ const. [5] solve this in the following way. For any desired vector $\mathbf{z}_{0}$ (which may be the uniform vector) with normalization $\mathbf{z}_{0}^{T} \mathbf{z}_{0}=1$, we can project the Green's matrix $\mathbf{G}$ onto the space orthogonal to $\mathbf{z}_{0}$,

$$
\mathbf{G}^{\prime}=\left(\mathbf{I}-\boldsymbol{z}_{0} \boldsymbol{z}_{0}^{T}\right) \mathbf{G}\left(\mathbf{I}-\boldsymbol{z}_{0} \boldsymbol{z}_{0}^{T}\right),
$$

and we use $\mathbf{G}^{\prime}$ instead of $\mathbf{G}$ in Eq. (3.8) to generate an eigenbasis. By construction, $\mathbf{G}^{\prime} \mathbf{z}_{0}=0$, and so $\mathbf{z}_{0}$ is an eigenvector of $\mathbf{G}^{\prime}$ with eigenvalue $\lambda^{-1}=0$.

\subsection{The Cryobasis}

We now detail how to create your own basis (the cryobasis for an arbirtrary domain $D$ ). Eq. (3.8) needs to be solved for its eigenvalues and eigenvectors. The spectrum of eigenvectors forms a matrix $\mathbf{Z}$ with each column an eigenvector $\mathbf{z}$. 

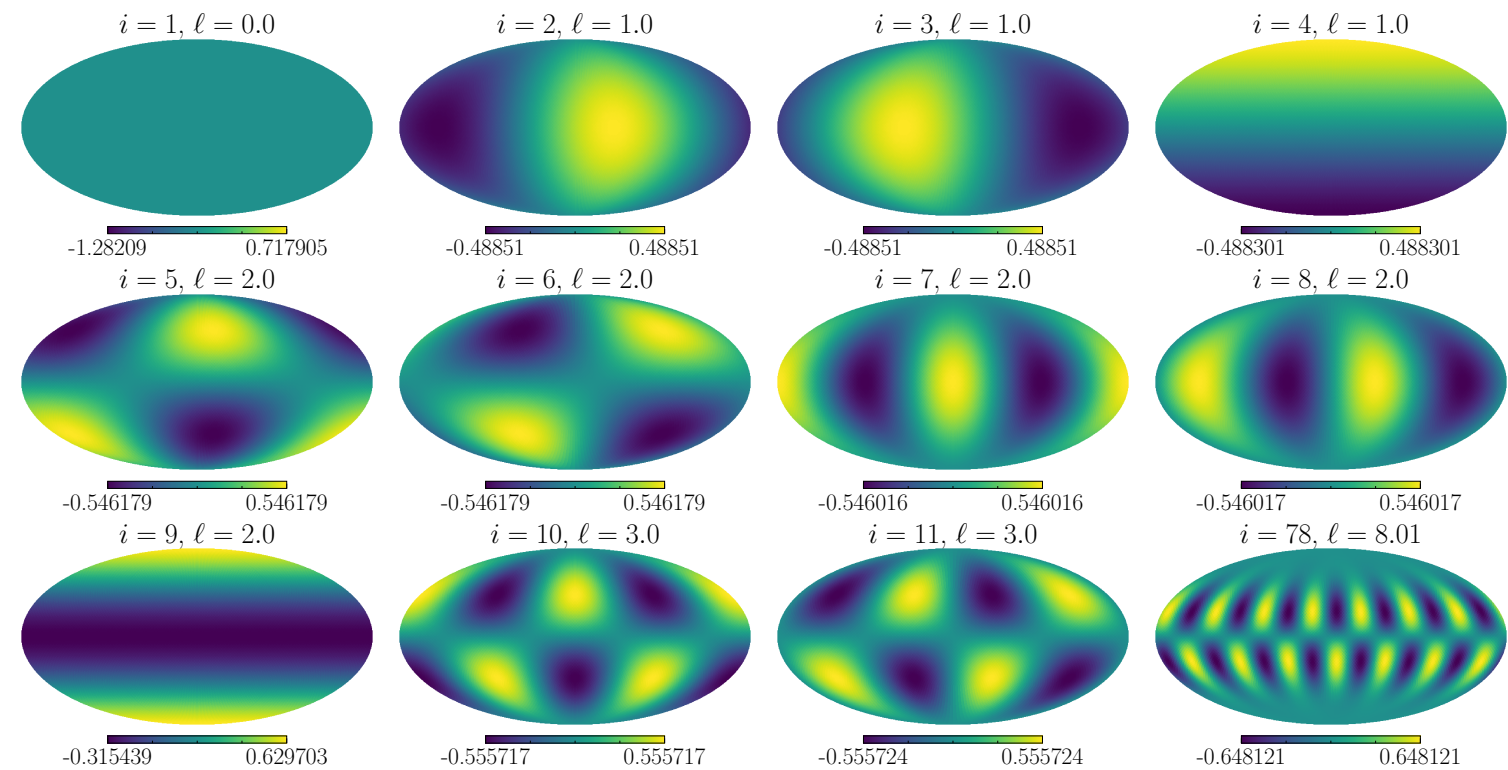

Figure 1. The first few $2 \mathrm{D}$ cryofunktions for a scalar field on the full sky at resolution $n_{\text {side }}=32$. The index $i$ simply enumerates the cryofunktions. There are $2 \ell+1$ cryofunktions at each $\ell$, as expected, and they are closely related to real spherical harmonics (see Eq. (A.3)).
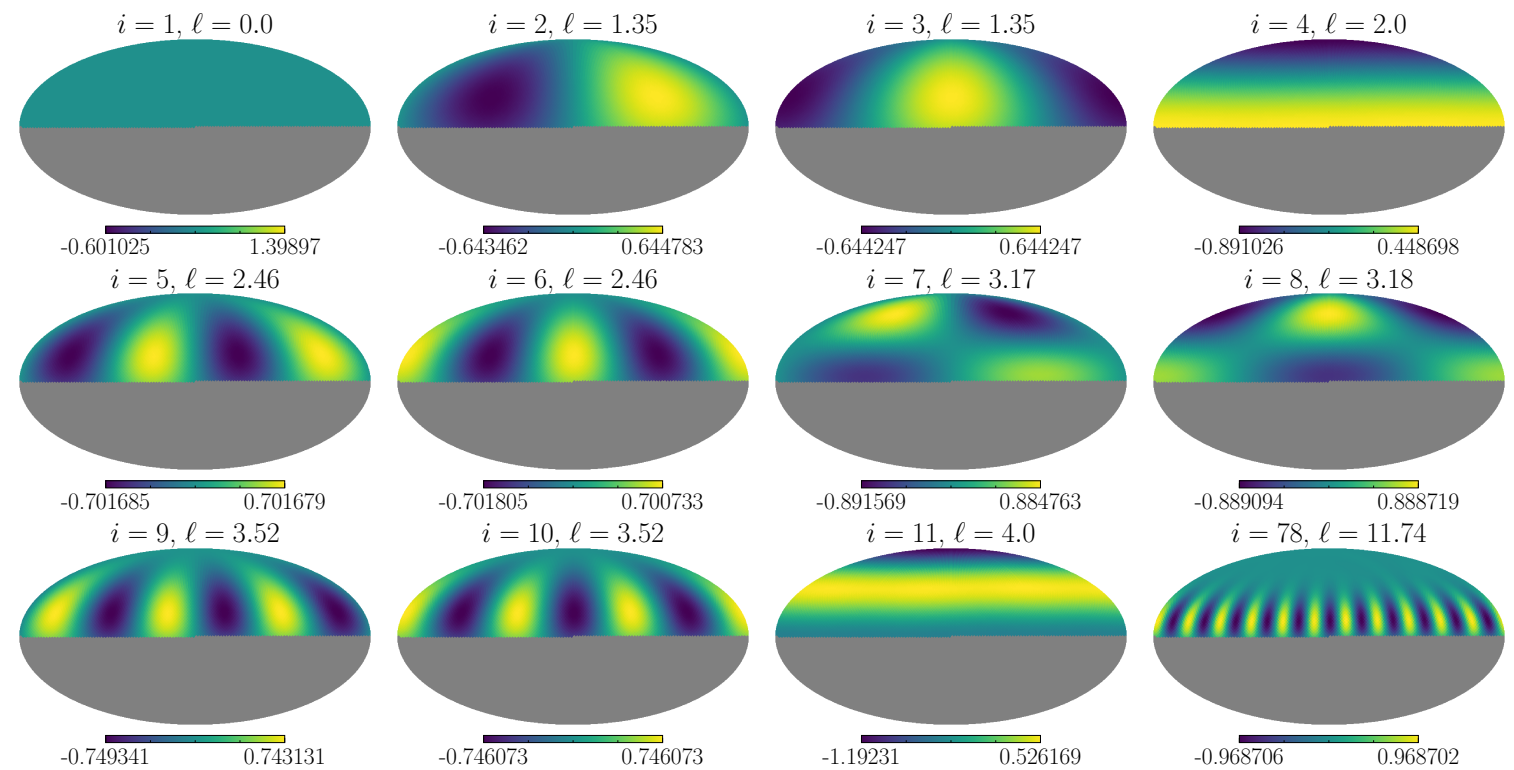

Figure 2. The $2 \mathrm{D}$ cryofunktions for a field on half the sky at resolution $n_{\text {side }}=32$. The greyed-out area is masked. Here, the effective $\ell$ is non-integer as each cryofunktion is a linear combination of spherical harmonics. 

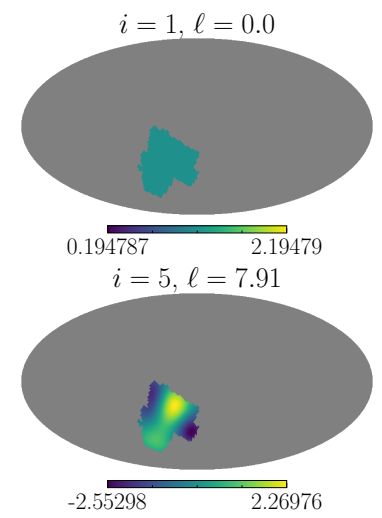

$i=9, \ell=10.99$

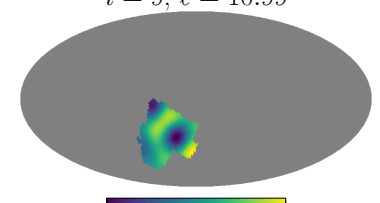

$-2.92404 \quad 2.41342$

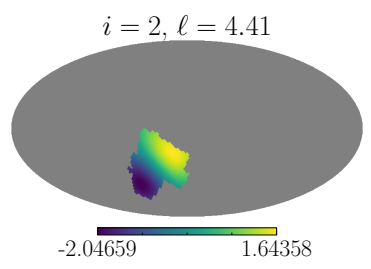

$i=6, \ell=8.04$

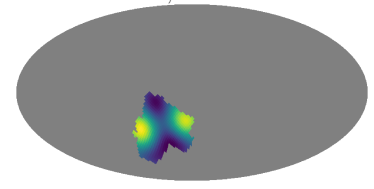

$- 1 . 7 \longdiv { 2 4 5 6 \quad 2 . 4 5 4 8 2 }$

$i=10, \ell=11.79$

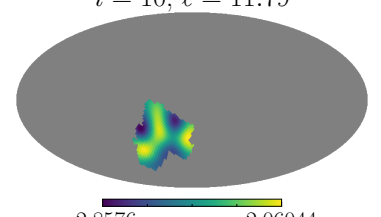

$-2.8 \overline{576 \quad 2.06044}$

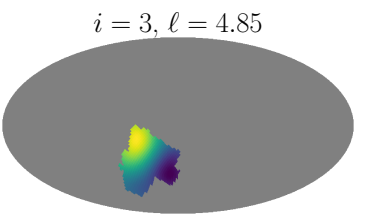

$-1.9 \overline{2542 \quad 2.0737}$

$i=7, \ell=9.87$

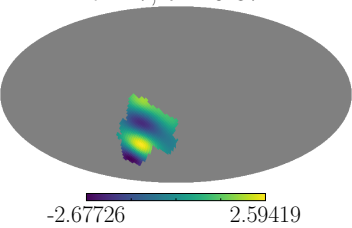

$i=11, \ell=12.49$

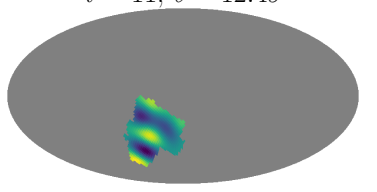

$-2.7236 \quad 2.76354$

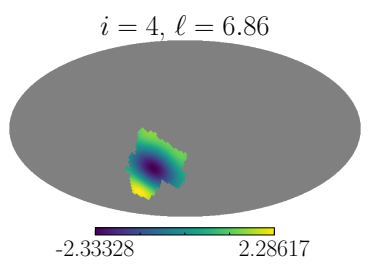

$i=8, \ell=10.47$

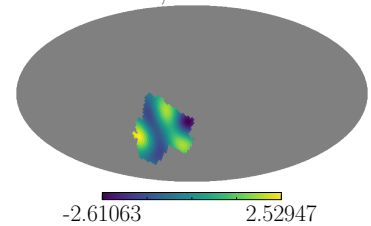

$i=78, \ell=36.68$

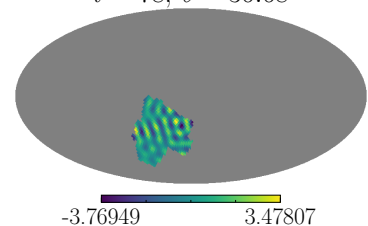

Figure 3. The $2 \mathrm{D}$ cryofunktions for a Roman-like angular mask at resolution $n_{\text {side }}=32$. For our smallest-sky-coverage example the cryomodes are sparse on large scales and quickly grow to larger $\ell$.
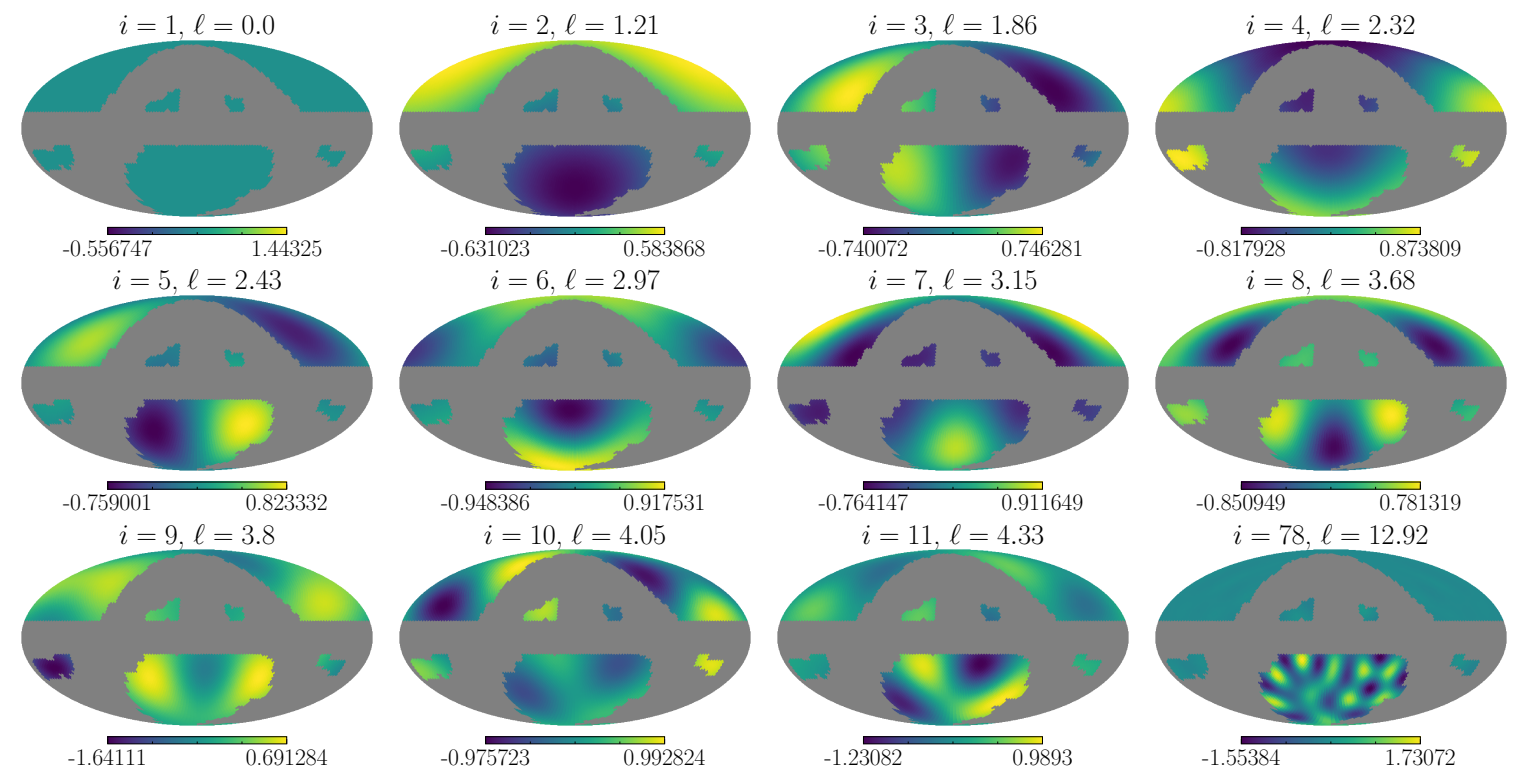

$i=7, \ell=3.15$

$i=8, \ell=3.68$
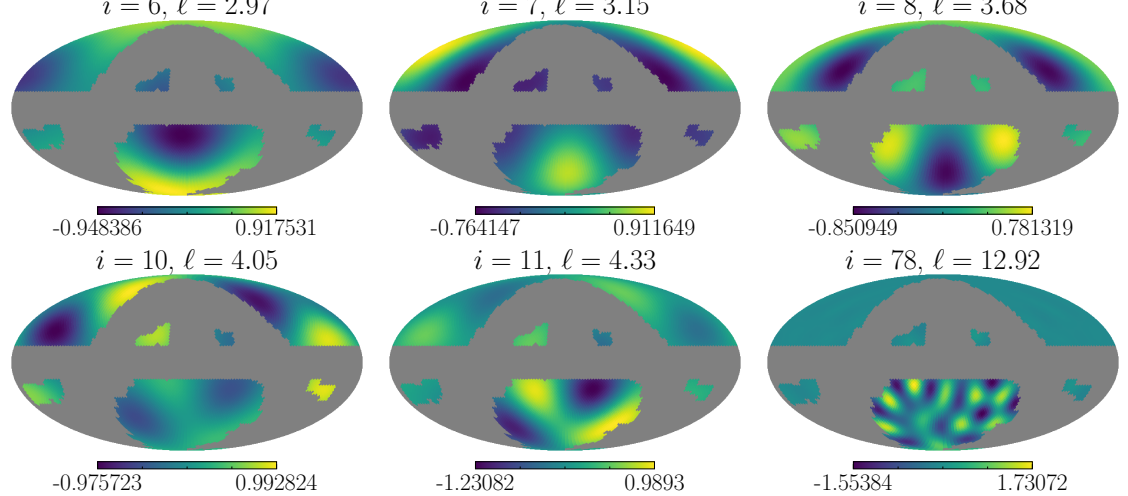

Figure 4. The $2 \mathrm{D}$ cryofunktions for a Euclid-like angular mask at resolution $n_{\text {side }}=32$. This mask is an example with multiple disconnected areas. 

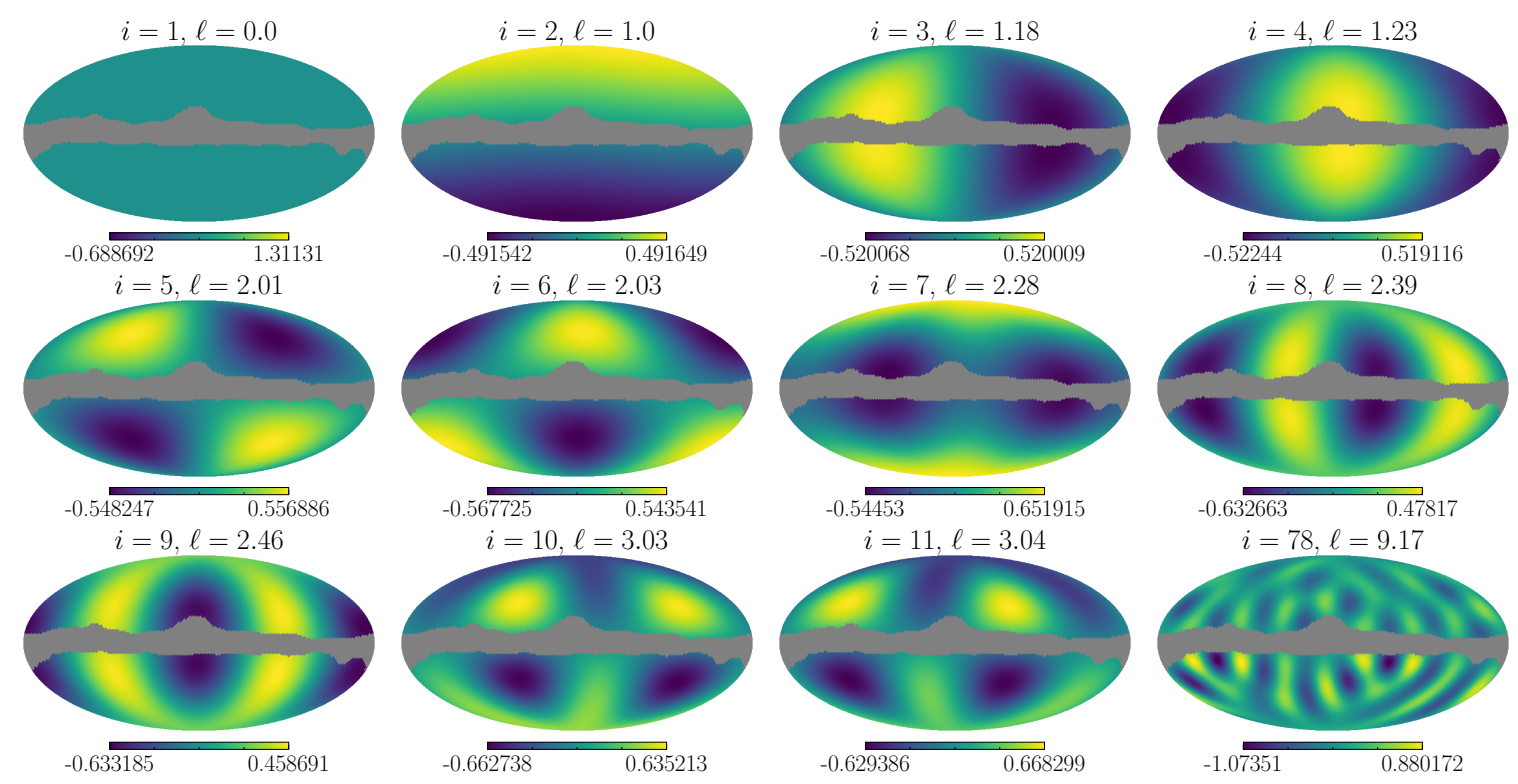

Figure 5. The 2D cryofunktions for a SPHEREx-like angular mask at resolution $n_{\text {side }}=32$. With a sky-coverage of $\sim 80 \%$, the cryofunktions for SPHEREx are closer to full-sky modes.

Since the construction of $\mathbf{z}$ in Eq. (3.5) involves factors of $\Omega_{i}^{s}$, the eigenvectors $\mathbf{y}$ that form the cryobasis are given by

$$
\mathbf{z}=\mathbf{B} \mathbf{y}
$$

where the transformation matrix $\mathbf{B}$ is diagonal with non-zero entries

$$
B_{i i}=\Omega_{i}^{s}
$$

With this definition, the entries in the vector $\mathbf{y}$ are the averages of the eigenfunction $Y$ over each pixel and we discuss the eigenvalues in the next section. The use of the HEALPix scheme allows the reduction of the matrix $\mathbf{B}$ to a scalar.

The symmetry of the Green's matrix $\mathbf{G}$ implies the relation

$$
\mathbf{Z}^{T} \mathbf{Z}=\mathbf{I}=\mathbf{Z} \mathbf{Z}^{T}
$$

Therefore,

$$
\mathbf{Y}^{T} \mathbf{B}^{T} \mathbf{B} \mathbf{Y}=\mathbf{I}=\mathbf{Y} \mathbf{Y}^{T} \mathbf{B}^{T} \mathbf{B}
$$

where $\mathbf{Y}$ is the matrix with vectors $\mathbf{y}$ as its columns, and the second equality follows from the second equality in Eq. (3.20) multiplied by $\mathbf{B}^{-1}$ from the left and $\mathbf{B}$ from the right. Thus, the inverse

$$
\mathbf{Y}^{-1}=\mathbf{Y}^{T} \mathbf{B}^{T} \mathbf{B}=\mathbf{Z}^{T} \mathbf{B}
$$

needs no explicit inverse, and, therefore, is very fast to calculate. This will be useful for the transform from configuration space to harmonic space. 

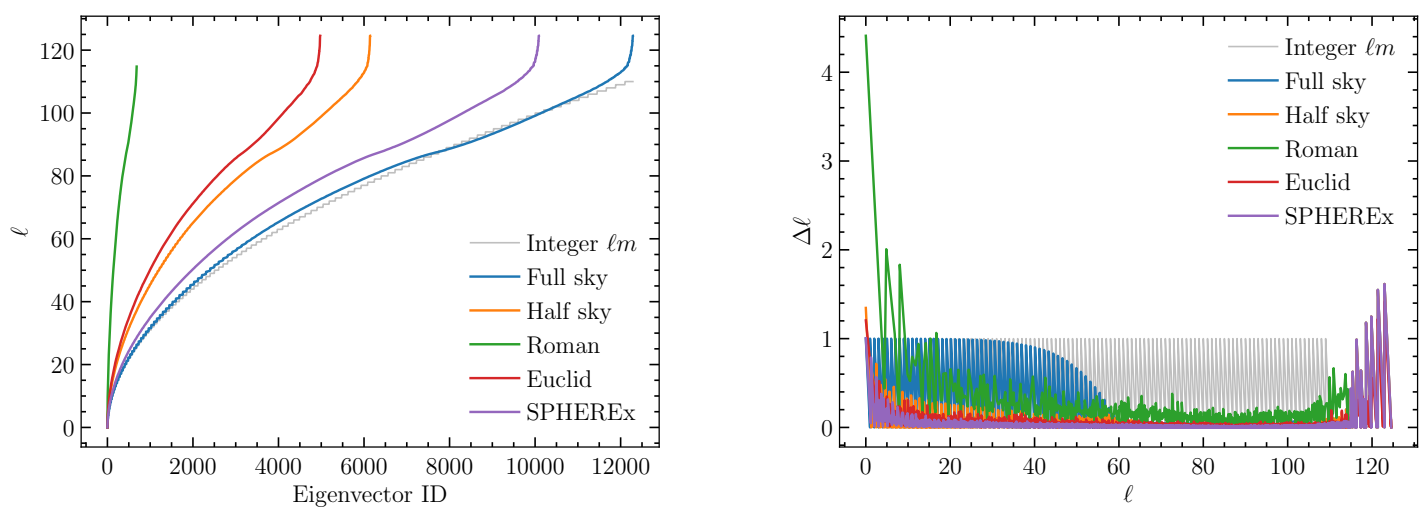

Figure 6. On the left we show the cryopoles at $n_{\text {side }}=32$ calculated for our five example masks as well as the standard full-sky-continuous basis (marked Integer $\ell m$ ). The number of eigenvectors is proportional the survey area. However, all surveys go to approximately the same maximum $\ell$. (However, only up to about $\ell \sim 2 n_{\text {side }}$ is usable, as shown in the text.) On the right we show the difference in the cryopole between neighboring eigenvectors. For the standard basis, each increment is either zero when $m$ increments or unity when $\ell$ increments. This holds true for the full-sky cryobasis for $\ell \lesssim n_{\text {side }}$ before taking on a wholly new form around $\ell \sim 2 n_{\text {side. }}$. None of the partial-sky cryobases follow such a structure.

Figs. 1-5 show the first few 2D cryofunktions over the sphere for several angular masks: full sky, half sky, Roman-like mask ${ }^{1}$, Euclid-like mask ${ }^{2}$, and SPHEREx-like mask ${ }^{3}$. Generally, for smaller sky fraction, there are fewer basis vectors that probe large scales.

\subsection{Cryopole transform}

With the basis in hand, we now work out the details of performing the transform. If the scalar field of interest is given by the pixel-vector $\mathbf{x}$, then the eigendecomposition $\mathbf{k}$ represents $\mathbf{x}$ in terms of the eigenvectors $\mathbf{Y}$. The harmonic transform pair is, then,

$$
\begin{aligned}
& \mathbf{x}=\mathbf{Y} \mathbf{k}, \\
& \mathbf{k}=\mathbf{Y}^{-1} \mathbf{x} .
\end{aligned}
$$

That is, the value of the scalar field in pixel $i$ is given by $x_{i}=\sum_{j} Y_{i j} k_{j}$, the linear combination of the contributions of each cryovector $\mathbf{y}_{j}$ to pixel $i$.

The eigenvalues (cryovalues) $\lambda^{-1}$ of $\mathbf{G}$ and $\mathbf{G}^{\prime}$ are the inverses of the eigenvalues $\lambda$ of the Laplace operator (see Eq. (3.8)),

$$
\lambda^{-1}=[\ell(\ell+1)]^{-1},
$$

where the multiplicity of the eigenvalue is $\sim 2 \ell+1$ for full-sky coverage. Due to the Hermiticity of the Green's matrix, these eigenvalues are real. Only the full-sky has integer-valued $\ell$, as shown at the top of each panel in Figs. 1-5. However, even for the full sky, the pixelization results in non-integer modes for $\ell \gtrsim n_{\text {side }}$.

\footnotetext{
${ }^{1}$ https://www.roman-hls-cosmology.space/, https://roman.gsfc.nasa.gov/

${ }^{2}$ https://www.euclid-ec.org/

${ }^{3}$ https://spherex.caltech.edu/
} 

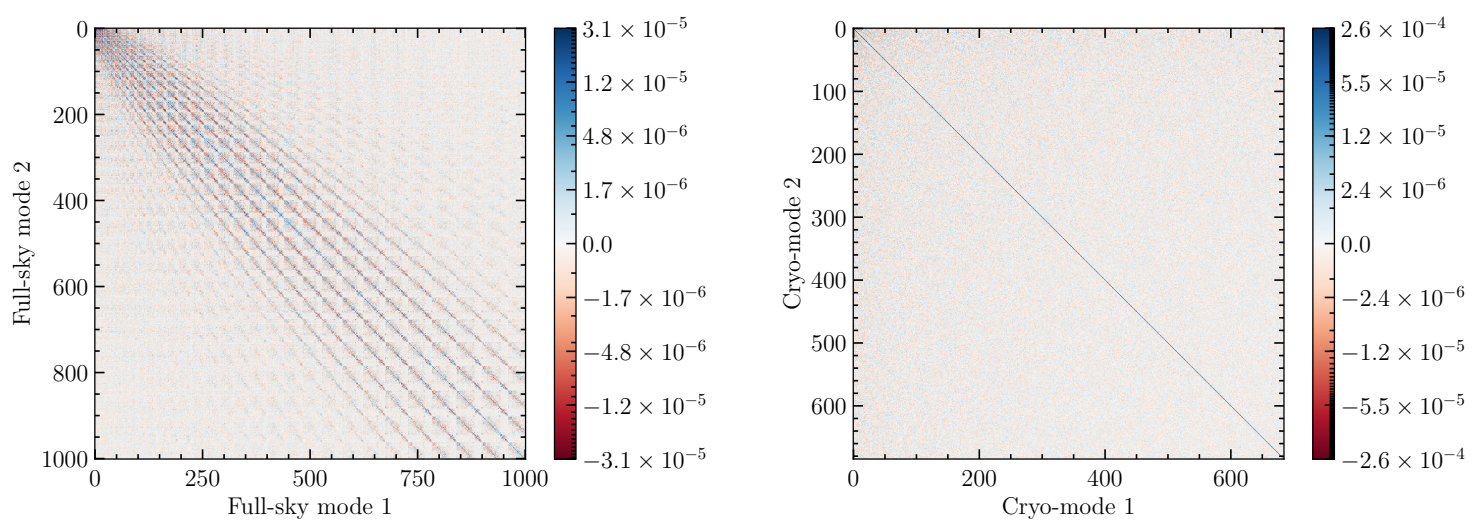

Figure 7. For Roman-like mask, the left panel shows $\mathbf{P}=\mathbf{k k}^{T}$ derived using full-sky eigenfunctions; on the right, the same is shown but using our cryofunctions derived in this paper. Using full-sky eigenfunctions as in the left plot leads to a poor compression of the data with a lot of off-diagonal terms. This is due to the mask breaking the isotropy on the sky. Using the cryofunctions, however, leads to all the information being compressed into the diagonal.

Note that the uniform cryovector has vanishing eigenvalue $\lambda^{-1}=0$, which according to Eq. (3.25) would, surprisingly, correspond to an infinite $\ell$. However, this is due to the construction of the Green's matrix in Section 3.3. Therefore, in this case we set $\ell$ explicitly to the monopole $\ell=0$.

The left panel of Fig. 6 shows the corresponding $\ell$-mode for each eigenvector for the same five masks as in Figs. 1-5. The figure shows that the number of eigenvectors scales with the sky coverage. More precisely, the total number of eigenvectors is equal to the number of pixels in the survey. Furthermore, even for the full sky, the pixelization scheme reduces the number of modes significantly compared to the infinite-resolution limit starting around $2 n_{\text {side }}$ in the figure. For smaller sky coverage fraction the loss of modes is more significant.

The right panel of Fig. 6 shows the increment of $\ell$ from one mode to the next as a function of $\ell$. In the standard case the increment either vanishes when only $m$ increases, or it changes by unity. However, due to the pixelization and boundaries, the increment can be much larger or smaller. Note how the full-sky mask is the only mask that adheres to the standard expectation, and only for $\ell \lesssim 2 n_{\text {side. }}$. The other masks tend to have jumps in $\ell$ more spread out.

\subsection{Cryopower}

Now we construct the power spectrum in our new basis. Formally, the power spectrum is constructed as

$$
\mathbf{P}=\mathbf{k} \mathbf{k}^{T}=\mathbf{Y}^{-1} \mathbf{x} \mathbf{x}^{T} \mathbf{Y}^{T,-1}
$$

where Eq. (3.24) was used. For a general basis, $\mathbf{P}$ is a $N_{\text {pix }} \times N_{\text {pix }}$ matrix. However, symmetries reduce the number of cross-correlations so that all information is compressed into the diagonal. Indeed, as we have shown in Section 2, this is precisely the reason for choosing a basis that is an eigenbasis of the Laplace operator. We demonstrate the diagonal nature of this matrix by averaging over $10^{5}$ lognormal simulations in Fig. 7 . 

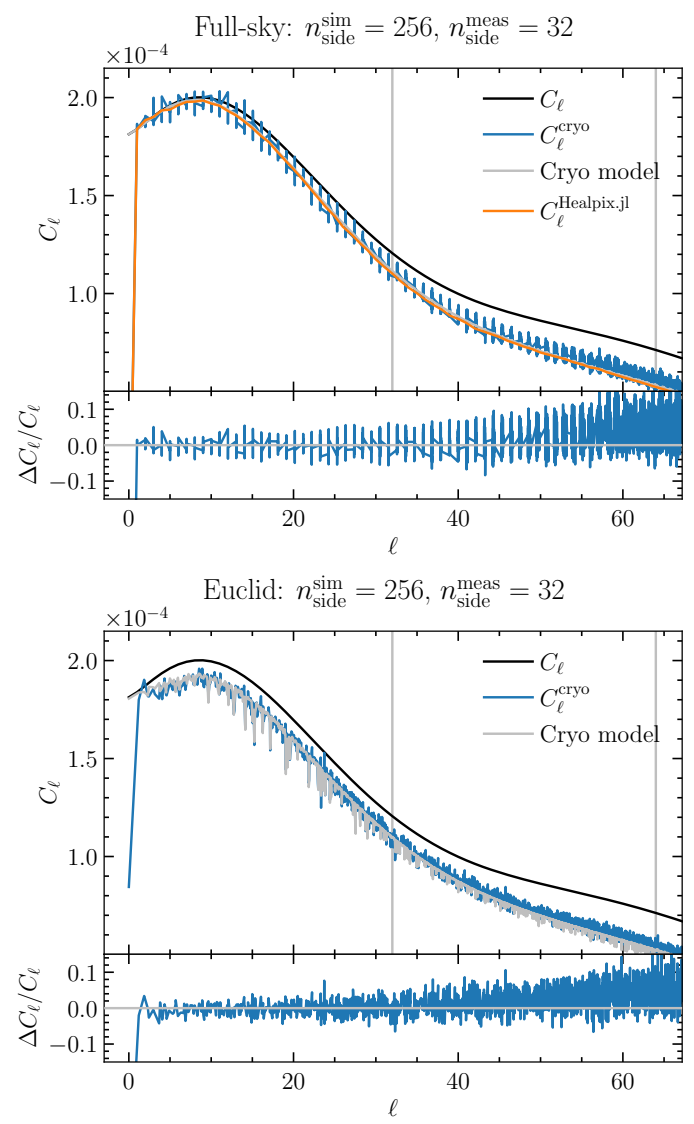
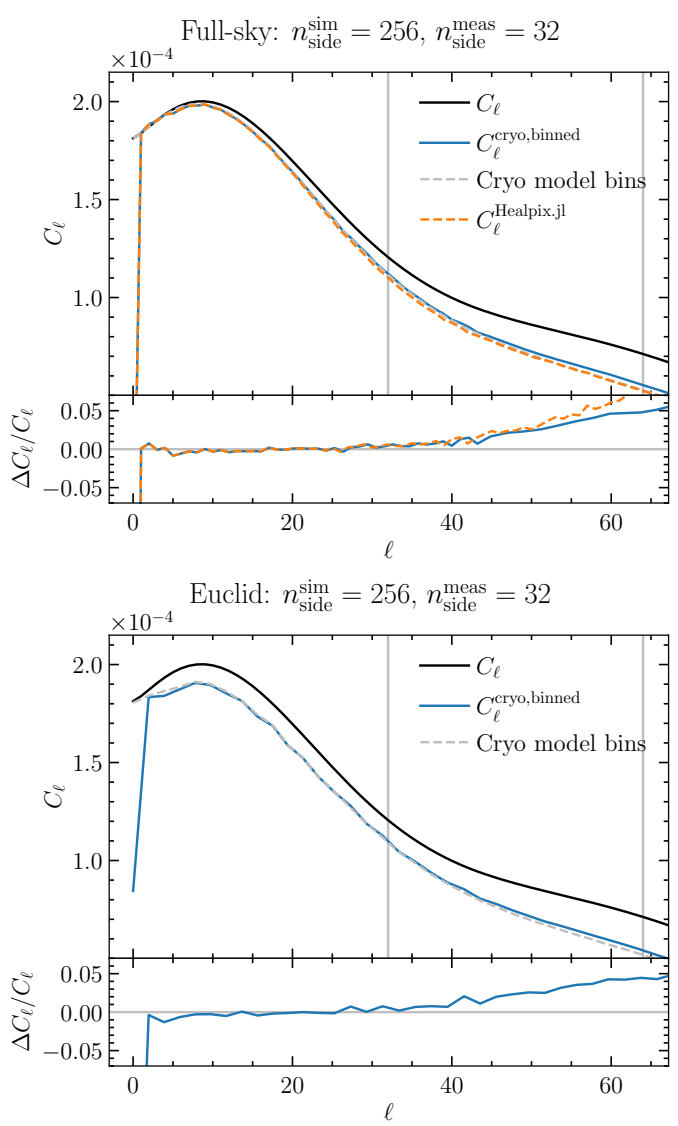

Figure 8. Top row: Full-sky cryospectrum without binning (left plot) and with binning modes with $\Delta \ell=1$ (right plot). Vertical grey lines are at integer multiples of $n_{\text {side. }}$ The black line are the input $C_{\ell}$, in blue is the measured cryo- $C_{\ell}$ averaged over $10^{4}$ simulations, grey is the model including cryo-window, and orange shows the result from HEALPix (without pixel window correction) for comparison. The lower plot in each panel shows the relative deviation between the estimated and modeled $C_{\ell}$. It shows a systematic bias above $\ell \gtrsim n_{\text {side }}$ that grows to $\sim 5 \%$ at $\ell \sim 2 n_{\text {side }}$. Bottom row: The same as the top row, but for a Euclid-like mask. Especially visible on the bottom left, the model traces the measured average including some downward spikes. The Euclid-like mask is the most challenging in this respect, and therefore it is the only one shown here. In all plots the $\ell=0$ mode is affected by the local average effect (or integral constraint).

The simulations were generated using a real-space galaxy $C_{\ell}\left(r, r^{\prime}\right)$ power spectrum [with the flat-sky approximation in 7] at $r \sim 1000 h^{-1} \mathrm{Mpc}$ with bin width $\Delta r=40 h^{-1} \mathrm{Mpc}$ and galaxy bias $b=1 / D(z)$, where $D(z)$ is the linear growth factor. We then used a similar procedure as in [8] to produce log-normal galaxy catalogs on the $2 \mathrm{D}$ sphere. We did not include redshift-space distortions, as our purpose was strictly to test the decomposition.

Crucial is to understand the pixel window and mask effects, which we turn to next.

\subsection{The cryo-window}

A crucial part of interpreting the cryo-measured power spectrum is its relation to the full-sky standard-spherical harmonic power spectrum. This requires modeling of both geometry (as part of the cryofunktions) and the pixel window. 
Consider two cryobases $\mathbf{Y}_{0}$ and $\mathbf{Y}_{1}$, where $\mathbf{Y}_{0}$ is the basis for a full sky, and $\mathbf{Y}_{1}$ for a particular survey mask. We then define the effect of the mask by linear transformation matrices $\mathbf{w}$ and $\widetilde{\mathbf{w}}$ such that

$$
\begin{aligned}
& \mathbf{x}_{1}=\mathbf{w x}_{0}, \\
& \mathbf{k}_{1}=\widetilde{\mathbf{w}} \mathbf{k}_{0} .
\end{aligned}
$$

If the full sky has $N_{\text {pix }}$ pixels, and the survey has $N_{\text {pix }}^{\text {survey }} \leq N_{\text {pix }}$ pixels, then the matrix w is $N_{\text {pix }}^{\text {survey }} \times N_{\text {pix }}$ and it selects which pixels from the full sky are observed by the survey. The matrix $\widetilde{\mathbf{w}}$ has the same size as $\mathbf{w}$, and can be obtained from the definition and the transform Eqs. (3.23) and (3.24),

$$
\mathbf{k}_{1}=\mathbf{Y}_{1}^{-1} \mathbf{x}_{1}=\mathbf{Y}_{1}^{-1} \mathbf{w} \mathbf{x}_{0}=\mathbf{Y}_{1}^{-1} \mathbf{w} \mathbf{Y}_{0} \mathbf{k}_{0}
$$

Hence,

$$
\widetilde{\mathbf{w}}=\mathbf{Y}_{1}^{-1} \mathbf{w} \mathbf{Y}_{0}
$$

By construction,

$$
\mathbf{w} \mathbf{w}^{T}=\mathbf{I},
$$

since $\mathbf{w} \mathbf{A} \mathbf{w}^{T}$ selects the rows and columns in the survey for any matrix $\mathbf{A}$, in particular $\mathbf{A}=\mathbf{I}$. Note that Eq. (3.22) then implies

$$
\widetilde{\mathbf{w}} \widetilde{\mathbf{w}}^{T}=\mathbf{I}, \quad \text { if } \mathbf{B}=\alpha \mathbf{I}
$$

for some scalar $\alpha$ that is the same for both the full sky and partial sky.

Then, the power spectra measured from two bases $\mathbf{Y}_{0}$ and $\mathbf{Y}_{1}$ are related by

$$
\mathbf{P}_{1}=\widetilde{\mathbf{w}} \mathbf{P}_{0} \widetilde{\mathbf{w}}^{T}
$$

The matrix $\widetilde{\mathbf{w}}$ contains both the effect of the pixelization operation as well as the effect of the survey geometry. However, by virtue of using eigenfunctions to the Laplacian, both $\mathbf{P}_{1}$ and $\mathbf{P}_{0}$ are statistically diagonal, as we have showed explicitly for a Roman-like mask in Fig. 7.

\subsubsection{Continuous bases}

In practice, theoretical predictions will assume a continuous sky, or, $n_{\text {side }} \rightarrow \infty$. Only in this limit is no information lost. Therefore, in this subsection we take the $\mathbf{Y}_{0}$ basis to be precisely this continuous limit for a full-sky survey. The result is usually referred to as the pixel window correction ${ }^{4}$.

Eq. (3.33) relates the measured power spectrum using two different bases $\mathbf{Y}_{0}$ and $\mathbf{Y}_{1}$. Nowhere have we assumed that these two bases have a common origin (such as being eigenbases of the Laplacian). In the limit $n_{\text {side }} \rightarrow \infty$ for the $Y_{0}$ base, the key difference is that we now have the operator

$$
\mathbf{w} \rightarrow \int \mathrm{d}^{2} \hat{\boldsymbol{r}} w_{i}(\hat{\boldsymbol{r}})
$$

\footnotetext{
${ }^{4}$ https://healpix.jpl.nasa.gov/html/intronode14.htm
} 
To be explicit, the transform from harmonic to configuration space for the continuous $\mathbf{Y}_{0}$ basis is

$$
\delta_{0}(\hat{\boldsymbol{r}})=\sum_{k} Y_{k}(\hat{\boldsymbol{r}}) \widetilde{\delta}_{0, k}
$$

where $k$ indexes the $\ell m$ modes, and for clarity we leave off the suffix 0 on $Y_{k}$. The pixelization scheme (for the $\mathbf{Y}_{1}$ basis) gives pixel values

$$
\delta_{1, j}=\int \mathrm{d}^{2} \hat{\boldsymbol{r}} w_{j}(\hat{\boldsymbol{r}}) \delta_{0}(\hat{\boldsymbol{r}}),
$$

and the transform

$$
\widetilde{\delta}_{1, i}=\sum_{j} Y_{1, i j}^{-1} \delta_{1, j}
$$

Then, the harmonic decompositions in the two bases are related by

$$
\widetilde{\delta}_{1, i}=\sum_{k} \widetilde{w}_{i k} \widetilde{\delta}_{0, k}
$$

where the transformation matrix is

$$
\widetilde{w}_{i k}=\sum_{j} Y_{1, i j}^{-1} \int \mathrm{d}^{2} \hat{\boldsymbol{r}} w_{j}(\hat{\boldsymbol{r}}) Y_{k}(\hat{\boldsymbol{r}}) .
$$

The indices $i$ and $j$ run from one to $N_{\text {pix }}^{\text {survey }}$, and the index $k$ runs over infinitely many $\ell m$ modes. In practice, of course, the sum needs to be truncated and only a finite number of modes are kept.

Clearly, the challenge is in calculating the integral in Eq. (3.39), which is the pixel window for a full-sky survey. Explicitly writing $k=\ell m$, that integral is

$$
w_{\ell m}(j)=\int \mathrm{d}^{2} \hat{\boldsymbol{r}} w_{j}(\hat{\boldsymbol{r}}) Y_{\ell m}(\hat{\boldsymbol{r}}) .
$$

A well-established approximation for HEALPix is to write

$$
w_{\ell m}(j) \simeq w_{\ell}(j) Y_{\ell m}(j),
$$

where $Y_{\ell m}(j)$ is typically evaluated at the center of pixel $j$. For $Y_{\ell m}(j)$ we take the real spherical harmonics Eq. (A.3). Then,

$$
\widetilde{w}_{i, \ell m}=w_{\ell} \sum_{j} Y_{1, i j}^{-1} Y_{\ell m}(j),
$$

where we assume that $w_{\ell}$ is independent of the pixel $j$.

The power spectra are related by

$$
\left\langle\widetilde{\delta}_{1, m} \widetilde{\delta}_{1, n}^{*}\right\rangle=\sum_{k s} \widetilde{w}_{m k} \widetilde{w}_{n s}^{*}\left\langle\widetilde{\delta}_{0, k} \widetilde{\delta}_{0, s}^{*}\right\rangle .
$$


Next, we assume that only the diagonal of $\mathbf{k k}^{T}$ contains information due to the isotropy of the sky. Indeed, since we use the Laplacian we know that $\mathbf{P}_{1}$ is statistically diagonal, and we write

$$
C_{n}^{1, \mathrm{obs}}=\sum_{k}\left|\widetilde{w}_{n k}\right|^{2} C_{k}
$$

In terms of $\ell$-modes,

$$
C_{n}^{1, \mathrm{obs}}=\sum_{\ell} C_{\ell} \sum_{m}\left|\widetilde{w}_{n, \ell m}\right|^{2}
$$

An example $\widetilde{H}_{\lambda}^{\ell}=\sum_{m}\left|\widetilde{w}_{\lambda \ell}\right|^{2}$ transformation matrix is shown below in the left of Fig. 10.

We demonstrate our cryo-window calculation in the full-sky and for a Euclid-mask in Fig. 8. The model is sufficiently complicated that an inversion is nontrivial except for the full sky.

Note that in the bottom panels of Fig. 8 the power is suppressed on all scales. We attribute this to the cryo-window being a combination of pixel window and survey geometry effect.

\subsection{Shot noise}

The sampling of the density field by a limited number of points leads to a shot noise component in the power spectrum. To show that the shot noise is $1 / \bar{n}$ in the cryobasis, we use the equations from $[9,10]$, but for pixels on the sphere,

$$
\begin{aligned}
\left\langle n(\hat{\boldsymbol{r}}) n\left(\hat{\boldsymbol{r}}^{\prime}\right)\right\rangle & =\bar{n}(\hat{\boldsymbol{r}}) \bar{n}\left(\hat{\boldsymbol{r}}^{\prime}\right)\left[1+\xi\left(\hat{\boldsymbol{r}}, \hat{\boldsymbol{r}}^{\prime}\right)\right]+\bar{n}(\hat{\boldsymbol{r}}) \delta^{D}\left(\hat{\boldsymbol{r}}-\hat{\boldsymbol{r}}^{\prime}\right), \\
\left\langle n(\hat{\boldsymbol{r}}) n_{r}\left(\hat{\boldsymbol{r}}^{\prime}\right)\right\rangle & =\alpha^{-1} \bar{n}(\hat{\boldsymbol{r}}) \bar{n}\left(\hat{\boldsymbol{r}}^{\prime}\right) \\
\left\langle n_{r}(\hat{\boldsymbol{r}}) n_{r}\left(\hat{\boldsymbol{r}}^{\prime}\right)\right\rangle & =\alpha^{-2} \bar{n}(\hat{\boldsymbol{r}}) \bar{n}\left(\hat{\boldsymbol{r}}^{\prime}\right)+\alpha^{-1} \bar{n}(\hat{\boldsymbol{r}}) \delta^{D}\left(\hat{\boldsymbol{r}}-\hat{\boldsymbol{r}}^{\prime}\right) .
\end{aligned}
$$

The number density of galaxies per solid angle is

$$
n(\hat{\boldsymbol{r}})=\sum_{i} \delta^{D}\left(\hat{\boldsymbol{r}}-\hat{\boldsymbol{r}}_{i}\right)
$$

where $\hat{\boldsymbol{r}}_{i}$ is the position of galaxy $i$.

Now, the density contrast on the sphere is dependent on the galaxy-assignment scheme. We use the nearest-grid-point scheme (NGP). Thus, the number density of galaxies in pixel $i$ is

$$
n_{i}=\int \mathrm{d}^{2} \hat{r} w_{i}(\hat{\boldsymbol{r}}) n(\hat{\boldsymbol{r}})
$$

where $w_{i}(\hat{\boldsymbol{r}})$ is non-zero inside the pixel and vanishes elsewhere. We normalize $w_{i}(\hat{\boldsymbol{r}})$ by the pixel solid angle such that

$$
1=\int \mathrm{d}^{2} \hat{r} w_{i}(\hat{\boldsymbol{r}})
$$

The density contrast in pixel $i$ is now

$$
\delta_{i}=\frac{n_{i}-\alpha n_{r, i}}{\bar{n}}=\int \mathrm{d}^{2} \hat{r} w_{i}(\hat{\boldsymbol{r}})\left[\frac{n(\hat{\boldsymbol{r}})-\alpha n_{r}(\hat{\boldsymbol{r}})}{\bar{n}}\right],
$$


where $\bar{n}$ is the average number density of galaxies in a pixel, $n_{r}(\hat{\boldsymbol{r}})$ is the number of galaxies in a pixel for a random catalogue, and $\alpha$ adjusts the random catalogue size to the survey catalogue size. Hence, the correlation function for pixels $i$ and $j$ is

$\xi_{i j}=\frac{1}{\bar{n}^{2}} \int \mathrm{d}^{2} \hat{r} w_{i}(\hat{\boldsymbol{r}}) \int \mathrm{d}^{2} \hat{r}^{\prime} w_{j}\left(\hat{\boldsymbol{r}}^{\prime}\right)\left\langle n(\hat{\boldsymbol{r}}) n\left(\hat{\boldsymbol{r}}^{\prime}\right)-\alpha n_{r}(\hat{\boldsymbol{r}}) n\left(\hat{\boldsymbol{r}}^{\prime}\right)-\alpha n_{r}\left(\hat{\boldsymbol{r}}^{\prime}\right) n(\hat{\boldsymbol{r}})+\alpha^{2} n_{r}(\hat{\boldsymbol{r}}) n_{r}\left(\hat{\boldsymbol{r}}^{\prime}\right)\right\rangle$.

With Eqs. (3.46)-(3.48) we get

$$
\xi_{i j}=\frac{1}{\bar{n}^{2}} \int \mathrm{d}^{2} \hat{r} w_{i}(\hat{\boldsymbol{r}}) \int \mathrm{d}^{2} \hat{r}^{\prime} w_{j}\left(\hat{\boldsymbol{r}}^{\prime}\right)\left[\bar{n}(\hat{\boldsymbol{r}}) \bar{n}\left(\hat{\boldsymbol{r}}^{\prime}\right) \xi\left(\hat{\boldsymbol{r}}, \hat{\boldsymbol{r}}^{\prime}\right)+(1+\alpha) \bar{n}(\hat{\boldsymbol{r}}) \delta^{D}\left(\hat{\boldsymbol{r}}-\hat{\boldsymbol{r}}^{\prime}\right)\right] .
$$

As a simplification, we assume $\bar{n}(\hat{\boldsymbol{r}})=\bar{n}$ is the same in each observed pixel. (That is, we consider binary masks only.) Further, we assume an infinitely large random sample, or $\alpha \rightarrow 0$. Then,

$$
\xi_{i j}=\int \mathrm{d}^{2} \hat{r} w_{i}(\hat{\boldsymbol{r}}) \int \mathrm{d}^{2} \hat{r}^{\prime} w_{j}\left(\hat{\boldsymbol{r}}^{\prime}\right) \xi\left(\hat{\boldsymbol{r}}, \hat{\boldsymbol{r}}^{\prime}\right)+\frac{1}{\bar{n}} \int \mathrm{d}^{2} \hat{r} w_{i}(\hat{\boldsymbol{r}}) w_{j}(\hat{\boldsymbol{r}})
$$

Further, assuming pixels don't overlap and have area $\Omega_{i}$,

$$
\xi_{i j}=\int \mathrm{d}^{2} \hat{r} w_{i}(\hat{\boldsymbol{r}}) \int \mathrm{d}^{2} \hat{r}^{\prime} w_{j}\left(\hat{\boldsymbol{r}}^{\prime}\right) \xi\left(\hat{\boldsymbol{r}}, \hat{\boldsymbol{r}}^{\prime}\right)+\frac{\delta_{i j}^{K}}{\bar{n} \Omega_{i}} .
$$

Transforming into cryo-space just the shot noise,

$$
\begin{aligned}
N_{\lambda \lambda^{\prime}}^{\text {shot }} & =\frac{1}{\bar{n} \Omega_{i}} \sum_{i j} Y_{\lambda, i}^{-1} Y_{\lambda^{\prime}, j}^{*,-1} \delta_{i j}^{K}=\frac{1}{\bar{n} \Omega_{i}}\left(\mathbf{Y}^{-1} \mathbf{Y}^{-1, T}\right)_{\lambda \lambda^{\prime}} \\
& =\frac{\delta_{\lambda \lambda^{\prime}}^{K}}{\bar{n}}
\end{aligned}
$$

where we used the results from Section 3.4. In Fig. 8 the shot noise has been subtracted.

\section{Spherical Fourier-Bessel decomposition}

In this section we extend the cryomethod to a 3D survey geometry. In principle, any survey geometry could be accommodated. However, the computational complexity rapidly increases with the number of voxels ${ }^{5}$.

Furthermore, since, e.g., growth of structure will destroy the full 3D translational symmetry, it is desirable to assume isotropy on the sky, only. To achieve this, we write down the Laplacian in spherical coordinates,

$$
\nabla^{2} f=\frac{1}{r^{2}} \frac{\partial}{\partial r}\left(r^{2} \frac{\partial f}{\partial r}\right)+\frac{1}{r^{2} \sin \theta} \frac{\partial}{\partial \theta}\left(\sin \theta \frac{\partial f}{\partial \theta}\right)+\frac{1}{r^{2} \sin ^{2} \theta} \frac{\partial^{2} f}{\partial \phi^{2}}
$$

The eigenfunctions of the Laplacian are separable. That is, the eigenfunctions are composed of radial and angular eigenfunctions. For a full sky the angular eigenfunctions are the $Y_{\ell m}(\hat{\boldsymbol{r}})$ with eigenvalue $\ell(\ell+1)$, and Section 3 dealt with our angular cryofunctions for partial skies.

\footnotetext{
${ }^{5}$ We use the term voxel to refer to cells in $3 \mathrm{D}$ space.
} 
The radial basis for an infinite flat universe are the spherical Bessels $j_{\ell}(k r)$, and we turn to the cryo-version of these in the sections that follow.

Using the eigenfunctions to Eq. (4.1) allows exploitation of the isotropy on the sky, while keeping the radial decomposition as a separate problem. The transform is known as the spherical Fourier-Bessel (SFB) transform. Compared to a more standard Yamamotoestimator [11-13] where a single line of sight for each pair of galaxies is chosen, the SFB transform allows each individual galaxy its own line of sight. Therefore, wide-angle effects are fully modeled in this approach, and evolution with redshift can be accounted for as well. In a previous paper we have developed an estimator for the SFB power spectrum, SuperFaB, where we explicitly modeled the window function and only retained modes in a pseudo- $C_{\ell}$ fashion [2]. In this paper, we choose such SFB-like approach because it makes the exploitation of the isotropic symmetry simple for window functions that are separable into an angular mask and radial selection function, and the cryofunks will allow the full information of the 2-point function to be contained in the pseudo- $C_{\ell}$ estimator.

The SFB power spectrum depends not just on the overall wavenumber $k$, but also on the angular quantum number $\ell$, and this is important to distinguish radial and angular modes. A full-volume eigendecomposition would, in general, leave us with indeterminate $\ell$. Therefore, we here opt to explicitly separate angular and radial modes. However, this means that the survey geometry must look the same for every sight line. More specifically, we require that the window can be written as $W(\boldsymbol{r})=\phi(r) M(\hat{\boldsymbol{r}})$ for some radial selection $\phi(r)$ and angular window $M(\hat{\boldsymbol{r}})$, and in this paper we further limit ourselves to binary selections. We call the resulting estimator $\mathrm{CryoFaB}$.

\subsection{Radial Modes}

The angular eigenfunctions influence the radial eigenfunctions because the angular mask will generally lead to non-integer $\ell$ and, hence, to spherical Bessel functions of non-integer order. Therefore, we first derive the 2D angular cryofunk basis as in Section 3, then we propagate the $\ell$ down to the radial basis functions.

The full Laplacian is shown in Eq. (4.1). The angular part was treated in Section 3. To treat the radial part, we first write an eigenfunction as $f_{\ell}\left(k_{n \ell} ; \boldsymbol{r}\right)=g_{n \ell}(r) Y_{\ell}(\hat{\boldsymbol{r}})$ (with $m$-dependency implicit in $\ell$, and $n$ enumerating $k_{n \ell}$-modes), then the angular part of the Laplacian acts on $Y_{\ell}(\hat{\boldsymbol{r}})$ only, and what is left is the radial part of the Laplacian acting on the radial eigenfunction $g_{n \ell}(r)$,

$$
\nabla_{r}^{2}=\frac{1}{r^{2}}\left[\frac{\mathrm{d}}{\mathrm{d} r}\left(r^{2} \frac{\mathrm{d}}{\mathrm{d} r}\right)-\ell(\ell+1)\right]
$$

and the differential equation for the radial eigenfunctions is

$$
\nabla_{r}^{2} g_{n \ell}(r)=-k_{n \ell}^{2} g_{n \ell}(r),
$$

where $-k_{n \ell}^{2}$ are the eigenvalues. The Green's function satisfies

$$
\nabla_{r}^{2} G\left(r, r^{\prime}\right)=-\delta^{D}\left(r^{\prime}-r\right)
$$

with solution

$$
G\left(r, r^{\prime}\right)= \begin{cases}\frac{r^{\prime}}{2 \ell+1}\left(\frac{r}{r^{\prime}}\right)^{\ell} & \text { for } r \leq r^{\prime} \\ \frac{r^{\prime}}{2 \ell+1}\left(\frac{r}{r^{\prime}}\right)^{-\ell-1} & \text { for } r^{\prime} \leq r\end{cases}
$$



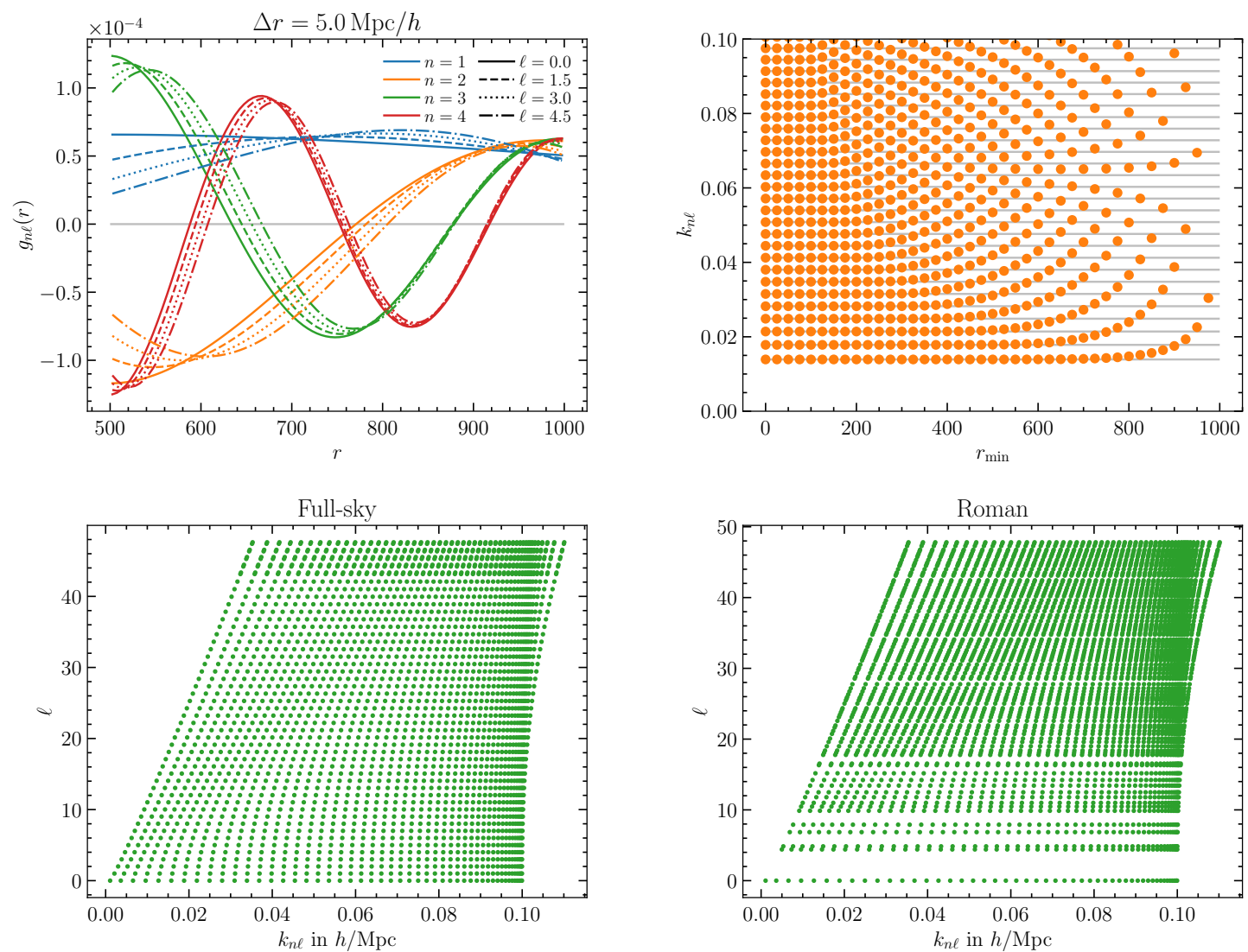

Figure 9. The top left plot shows some radial eigenfunctions of the inverse Laplacian, and the top right shows the $k_{n \ell}$-modes as a function of $r_{\min }$ at $\ell=10$. Slight discrepancies with the SuperFab paper disappear as the number of radial bins is increased. In the bottom two panels we show all the radial modes for each angular mode $\ell$, for a full-sky survey (left panel) and a Roman-like mask (right panel). In these plots each dot represents the $k_{n \ell}$ for one $(n, \ell)$ combination. (However, to limit the total number of points, we do not plot more than one $\ell$ per $\Delta \ell=0.2$.)

Eq. (4.5) is not symmetric under exchange of $r$ and $r^{\prime}$. However, it can be written

$$
G\left(r, r^{\prime}\right)=r^{p-2} r^{\prime p} \widetilde{G}\left(r, r^{\prime}\right)
$$

Then, for any $p$, the kernel $\widetilde{G}\left(r, r^{\prime}\right)$ is symmetric. While symmetry is not required, it allows the use of more efficient algorithms for determining the eigenfunctions.

Using the Green's function to get radial solutions to the eigenequation Eq. (4.3), we get the integral equation

$$
g_{n \ell}(r)=k_{n \ell}^{2} \int \mathrm{d} r^{\prime} G\left(r, r^{\prime}\right) g_{n \ell}\left(r^{\prime}\right)
$$

Same as for the angular part, we discretize by introducing a pixel-averaged quantity

$$
z_{i}=\Delta r_{i}^{s} \int \mathrm{d} r r^{m} w_{i}(r) g_{n \ell}(r)
$$


where $w_{i}(r)=1 / \Delta r_{i}$ inside the pixel and zero outside, and we introduce a weight $r^{m}$ that will help symmetrize the Green's matrix. The approximation for $g_{n \ell}\left(r^{\prime}\right)$ inside the pixel $j$ is $\Delta r_{j}^{-s} r^{-m} z_{j}$. Then,

$$
z_{i} \simeq k^{2} \sum_{j} \Delta r_{i}^{s} \Delta r_{j}^{1-s} z_{j} \int \mathrm{d} r r^{p+m-2} w_{i}(r) \int \mathrm{d} r^{\prime} r^{\prime p-m} w_{j}\left(r^{\prime}\right) \widetilde{G}\left(r, r^{\prime}\right),
$$

which is the matrix equation

$$
\mathbf{z}=k^{2} \mathbf{G} \mathbf{z}
$$

where $\mathbf{G}$ has elements

$$
\begin{aligned}
G_{i j} & =\Delta r_{i}^{s} \Delta r_{j}^{1-s} \int \mathrm{d} r r^{p+m-2} w_{i}(r) \int \mathrm{d} r^{\prime} r^{\prime p-m} w_{j}\left(r^{\prime}\right) \widetilde{G}\left(r, r^{\prime}\right) . \\
& =\Delta r_{i}^{s} \Delta r_{j}^{1-s} \int \mathrm{d} r r^{m} w_{i}(r) \int \mathrm{d} r^{\prime} r^{\prime-m} w_{j}\left(r^{\prime}\right) G\left(r, r^{\prime}\right) .
\end{aligned}
$$

$G_{i j}$ is symmetric provided that $s=\frac{1}{2}$ and $m=1$. The Green's function Eq. (4.5) is welldefined everywhere except at $r=0$ or $r^{\prime}=0$. Therefore, we evaluate $G_{i j}$ by taking the center of each bin, or

$$
G_{i j} \simeq \Delta r_{i}^{s} \Delta r_{j}^{1-s} r_{i}^{m} r_{j}^{-m} G\left(r_{i}, r_{j}\right)
$$

Similar to the matrix $\mathbf{B}$ in Eq. (3.18) that relates the eigenfunction $\mathbf{z}$ of the symmetric $G_{i j}$ to the basis function of the Laplacian, there is a corresponding matrix B for the radial eigenfunctions. This matrix is diagonal with elements given by

$$
B_{i j}=\delta_{i j}^{K} \Delta r_{i}^{s} r_{i}^{m}
$$

The procedure outlined here gives for each angular $\ell$-mode a set of radial eigenfunctions with radial mode $k_{n \ell}$. The eigenfunctions are defined only up to an overall phase factor which we fix so that all eigenvectors have the same sign at $r_{\max }$. The first few eigenvectors are shown in Fig. 9. The exquisite agreement with [2, Fig. 1] shows that the boundary conditions are in this case essentially the same as in that paper, namely they are potential boundaries where the basis functions satisfy the Helmholtz equation Eq. (4.3) inside the survey and $\nabla_{\boldsymbol{r}}^{2} g_{n \ell}=0$ outside the survey and matching at the boundary [also see 14, Appendix A]. For a more formal discussion of the boundary conditions, see [4]. The first few basis functions are illustrated in Fig. 9, where we used radial bins of size $\Delta r=10 h^{-1} \mathrm{Mpc}$. The small differences to [2] vanish when decreasing the size of the bins.

The bottom panels of Fig. 9 show the combinations of $k_{n \ell}$ and $\ell$ for the full-sky (bottom left) and a Roman-like mask (bottom right), both for the same radial top-hat selection $500 h^{-1} \mathrm{Mpc} \leq r \leq 1500 h^{-1} \mathrm{Mpc}$. The figure shows that, as expected, higher- $\ell$ tend to probe smaller scales. Since $\ell$ probes the angular scale, and $k_{n \ell}$ the total physical scale, the modes with low $\ell$ near the left of the plot are primarily perpendicular to the line of sight, and modes near the right of the plot are primarily parallel to the line of sight. That is, for a given $\ell$ the modes become increasingly parallel as $k_{n \ell}$ increases. 

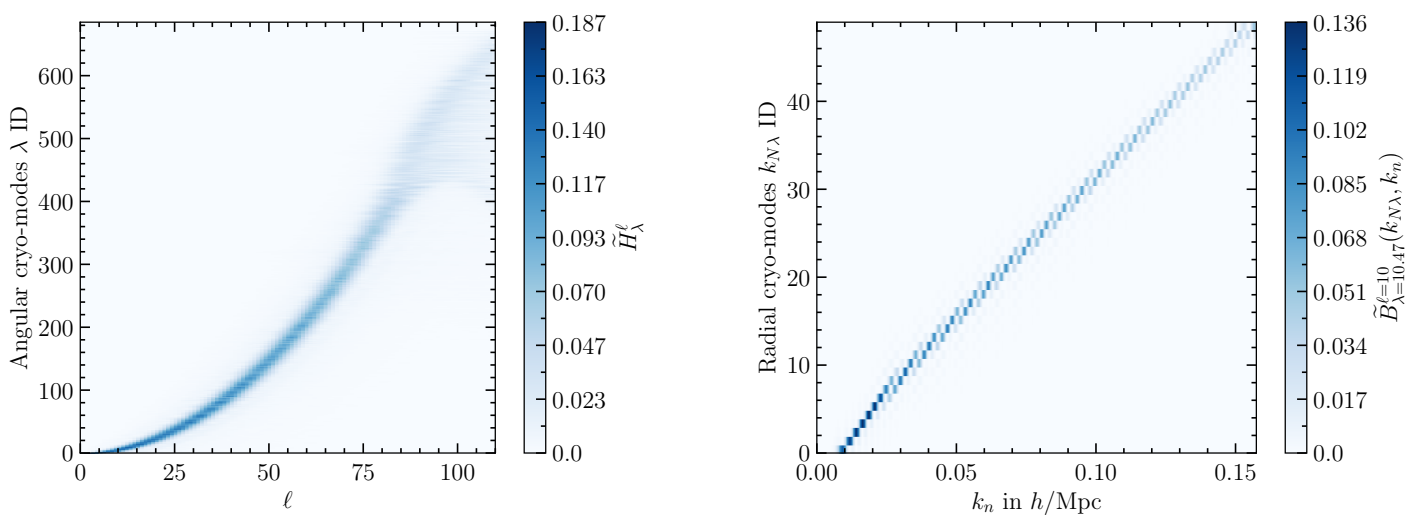

Figure 10. Left: Angular cryo-window for a Roman mask, Eq. (4.25). Right: Radial cryo-window for $\ell=10, \lambda=10.47$, Eq. (4.26). In both plots the ordinate is labeled with an eigenvector ID. The abscissa on the left shows full-sky continuous-resolution $\ell$-modes, while on the right it shows the $k$-modes. Note that the linearity on the right is an illusion: the cryo- $k$ on the ordinate are not linearly spaced, but become more dense at higher IDs, see the bottom-right panel in Fig. 9 for the precise values. In both plots we show the smaller scales (high cryovector ID) for completeness only.

\subsection{Into Cryospace}

In this section we explicitly show the full 3D transform. Since the eigenfunctions to the Laplacian can be separated into radial and angular parts, $f_{\ell}\left(k_{n \ell} ; \boldsymbol{r}\right)=g_{n \ell}(r) Y_{\ell}(\hat{\boldsymbol{r}})$, the full 3D transform from configuration space to cryo-space is

$$
\widetilde{\delta}_{n \ell}=\int \mathrm{d}^{3} r g_{n \ell}(r) Y_{\ell}(\hat{\boldsymbol{r}}) \delta(\boldsymbol{r}),
$$

(again, with degenerate $\ell$-modes implicitly labeled). Discretizing and performing the angular transform before the radial transform, we can write this as a matrix equation,

$$
\widetilde{\boldsymbol{\delta}}=\mathbf{R}^{-1} \mathbf{S}^{-1} \mathbf{A}^{-1} \boldsymbol{\delta},
$$

where $\mathbf{R}$ is the matrix of radial eigenfunctions, $\mathbf{A}$ are the angular eigenfunctions, and $\mathbf{S}$ is a matrix that reorders the elements in the data vector, and we introduce it for convenience of the implementation, as follows. If we first do the angular transform for each radial shell, then we can write this operation as a block diagonal matrix $\mathbf{A}$ acting on the data vector sorted into radial shells. Similarly, the radial transform can be written as a block diagonal matrix $\mathbf{R}$ on a data vector sorted into angular modes. Thus, the matrix $\mathbf{S}$ is needed to reshuffle the data vector so that both $\mathbf{R}$ and $\mathbf{A}$ can be block diagonal, each block operating on a single angular mode or single radial shell, respectively, and the full transform is given by Eq. (4.16).

\subsection{Cryo-Window}

In this section we calculate the combined effect of the pixel window and the geometry of the survey. The separation of radial and angular scales passes all the way through, starting with the assumption that the voxels are described by a separable binning function. That is, the configuration-space voxel window for radial bin $i$ and angular pixel $j$ is

$$
w_{i j}(\boldsymbol{r})=b_{i}(r) h_{j}(\hat{\boldsymbol{r}}),
$$


where $h_{j}(\hat{\boldsymbol{r}})$ specifies the $j$-th HEALPix pixel, and $b_{i}(r)$ is a top-hat specifying the $i$-th radial bin.

In the following we label the continuous-basis modes by $\delta_{\ell m}(k)$ and the pixelized cryomodes by $\delta_{N \lambda}$, where $k_{N \lambda}$ are the radial cryomodes and $\lambda$ are the angular cryomodes. That is, $\lambda=(\ell, m)$ is a combined index in case the $\ell$-modes are degenerate, and the doublet $(N, \lambda)$ in cryospace takes the role of the triplet $(k, \ell, m)$ in the full-sky continuous basis.

Transforming from continuous-limit harmonic-space density contrast to configuration space, then binning, and finally transforming into cryo-space yields

$$
\widetilde{\delta}_{N \lambda}^{1}=\sum_{i} R_{\lambda, N i}^{1,-1} \sum_{j} A_{\lambda j}^{1,-1} \int \mathrm{d} r b_{i}(r) \int \mathrm{d}^{2} \hat{r} h_{j}(\hat{\boldsymbol{r}}) \int \mathrm{d} k \sum_{\ell m} R_{\ell}^{0}(r, k) A_{\ell m}^{0}(\hat{\boldsymbol{r}}) \widetilde{\delta}_{\ell m}^{0}(k),
$$

where we attach the suffix 0 to indicate the full-sky continuous-limit basis, and the index 1 to indicate the pixelized finite-volume basis. That is, the harmonic-space pixelization operation is encoded in

$$
\widetilde{w}_{\lambda}^{\ell m}\left(k_{N \lambda}, k\right)=\widetilde{b}_{\lambda}^{\ell}\left(k_{N \lambda}, k\right) \widetilde{h}_{\lambda}^{\ell m},
$$

where we define the radial and angular harmonic-space pixelization matrices

$$
\begin{aligned}
\widetilde{b}_{\lambda}^{\ell}\left(k_{N \lambda}, k\right) & =\sum_{i} R_{\lambda, N i}^{1,-1} \int \mathrm{d} r b_{i}(r) R_{\ell}^{0}(r, k), \\
\widetilde{h}_{\lambda}^{\ell m} & =\sum_{j} A_{\lambda j}^{1,-1} \int \mathrm{d}^{2} \hat{r} h_{j}(\hat{\boldsymbol{r}}) \sum_{\ell m} A_{\ell m}^{0}(\hat{\boldsymbol{r}}) .
\end{aligned}
$$

Note that the second line is identical to Eq. (3.39).

The power spectrum in basis $i$ is, due to isotropy,

$$
\left\langle\widetilde{\delta}_{N \lambda}^{i} \widetilde{\delta}_{N^{\prime} \lambda^{\prime}}^{i, *}\right\rangle=\delta_{\lambda, \lambda^{\prime}}^{K} C_{\lambda N N^{\prime}}^{i}
$$

Therefore, the relation between two bases 0 and 1 corresponding to two distinct surveys and/or resolutions is

$$
C_{\lambda N N^{\prime}}^{1}=\int \mathrm{d} k \int \mathrm{d} k^{\prime} \sum_{\ell m} \widetilde{b}_{\lambda}^{\ell}\left(k_{N \lambda}, k\right) \widetilde{b}_{\lambda}^{\ell, *}\left(k_{N^{\prime} \lambda}, k^{\prime}\right)\left|\widetilde{h}_{\lambda}^{\ell m}\right|^{2} C_{\ell}^{0}\left(k, k^{\prime}\right) .
$$

In this proof-of-concept paper we only consider a radially homogeneous universe as our test case. We thus neglect the evolution along the line of sight due to, e.g., the growth of structure. Then, $C_{\ell}^{0}\left(k, k^{\prime}\right)=\delta^{D}\left(k-k^{\prime}\right) C_{\ell}^{0}(k)$ and $C_{\lambda N N^{\prime}}^{1}=\delta_{N N^{\prime}}^{K} C_{\lambda N}^{1}$, and Eq. (4.23) becomes

$$
C_{\lambda N}^{1}=\sum_{\ell} \sum_{n} \widetilde{H}_{\lambda}^{\ell} \widetilde{B}_{\lambda}^{\ell}\left(k_{N \lambda}, k_{n}\right) C_{\ell}^{0}\left(k_{n}\right),
$$

where the integral over $k$ was discretized into a sum over $n$, and we defined the angular and radial pixel functions

$$
\begin{aligned}
\widetilde{H}_{\lambda}^{\ell} & =\sum_{m}\left|\widetilde{h}_{\lambda}^{\ell m}\right|^{2} \\
\widetilde{B}_{\lambda}^{\ell}\left(k_{N \lambda}, k_{n}\right) & =\int_{k_{n}-\Delta k / 2}^{k_{n}+\Delta k / 2} \mathrm{~d} k\left|\widetilde{b}_{\lambda}^{\ell}\left(k_{N \lambda}, k\right)\right|^{2},
\end{aligned}
$$


assuming that $C_{\ell}^{0}(k)$ is constant across a step of size $\Delta k$. Both matrices $\widetilde{H}$ and $\widetilde{B}$ are sparse. $\widetilde{H}$ is peaked around $\ell \sim \lambda$, and $\widetilde{B}$ is sharply peaked around $k_{N \lambda} \sim k$, with some deviation for larger $k_{N \lambda}$. We show examples in Fig. 10.

\subsection{Local Average Effect}

Before we can compare estimator results with our analytical model, the issue of the local average effect, or integral constraint, must be addressed. In practice, the average number density $\bar{n}(z)$ must be measured from the survey itself, and this leads to the local average effect, which decreases the power in the $\ell=0$ mode. This is often called the integral constraint $[15,16]$ or the local average effect $[17,18]$. In this section, we detail the derivation.

Measuring the average number density as a function of redshift is accomplished by dividing the total number of galaxies in a redshift slice by the volume of that slice. That is, given the estimated number density $n(\hat{\boldsymbol{r}}, z)$, we get [also see, e.g., 19]

$$
\bar{n}(z)=\frac{1}{4 \pi f_{\text {sky }}} \int_{D} \mathrm{~d}^{2} \hat{r} n(\hat{\boldsymbol{r}}, z) .
$$

Relating this to the true average number density $\bar{n}^{\text {true }}$ (assumed constant), $n(\hat{\boldsymbol{r}}, z)=(1+\delta(\boldsymbol{r})) \bar{n}^{\text {true }}$, we get

$$
\bar{n}(z)=(1+\bar{\delta}(z)) \bar{n}^{\text {true }}
$$

where the average density contrast in the redshift slice at $z$ is

$$
\bar{\delta}(z)=\frac{1}{4 \pi f_{\text {sky }}} \int_{D} \mathrm{~d}^{2} \hat{r} \delta(\boldsymbol{r}) .
$$

Therefore, the estimated density contrast is

$$
\delta^{\mathrm{obs}}(\boldsymbol{r})=\frac{n(\hat{\boldsymbol{r}}, z)-\bar{n}(z)}{\bar{n}(z)}=\frac{\delta(\boldsymbol{r})-\bar{\delta}(z)}{1+\bar{\delta}(z)} \simeq \delta(\boldsymbol{r})-\bar{\delta}(z)
$$

where in the last line we assume a large volume so that $\bar{\delta}(z)$ is small. Compared to, e.g., [20], we have no explicit window function $W(\boldsymbol{r})$ as we are assuming a binary selection and mask in this paper, and $\boldsymbol{r}$ is understood to be within the survey.

Transforming into cryospace, the $\bar{\delta}(z)$ term only adds to the $\ell=0$ multipole. Hence, only $\ell=0$ modes are affected by the local average effect, or integral constraint.

Concretely, the transform is

$$
\begin{aligned}
\widetilde{\delta}_{n \lambda}^{\text {obs }} & =\sum_{i} R_{n i}^{\lambda,-1} \sum_{j} A_{\lambda j}^{-1}\left[\delta\left(\hat{\boldsymbol{r}}_{j}, z_{i}\right)-\bar{\delta}\left(z_{i}\right)\right] \\
& =\widetilde{\delta}_{n \lambda}-\sum_{i} R_{n i}^{\lambda,-1} \bar{\delta}\left(z_{i}\right) \sum_{j} A_{\lambda j}^{-1},
\end{aligned}
$$

where $i$ enumerates redshift bins, and $j$ enumerates angular pixels on the sphere. Define

$$
A_{\lambda} \equiv \sum_{j} A_{\lambda j}^{-1}
$$


Then, for a full-sky survey, $A_{\lambda}=\sum_{j} \Omega_{\text {pix }} Y_{\lambda m}^{*}\left(\hat{\boldsymbol{r}}_{j}\right) \simeq \sqrt{4 \pi} \delta_{\lambda 0}^{K} \delta_{m 0}^{K}$. More generally, for $\lambda=0$ we find that

$$
A_{j 0}=\frac{1}{\sqrt{4 \pi f_{\mathrm{sky}}}}, \quad A_{0 j}^{-1}=\frac{\Omega_{\mathrm{pix}}}{\sqrt{4 \pi f_{\mathrm{sky}}}}
$$

which follows from $A_{0 j}^{-1}=\Omega_{\mathrm{pix}} A_{j 0}$ and $\sum_{j} A_{0 j}^{-1} A_{j 0}=1$. Thus,

$$
A_{\lambda}=\frac{1}{\sqrt{4 \pi f_{\text {sky }}}} \frac{4 \pi N_{\text {pix }}^{\text {survey }}}{N_{\text {pix }}^{\text {full-sky }}} \delta_{\lambda 0}^{K}=\sqrt{4 \pi f_{\text {sky }}} \delta_{\lambda 0}^{K} .
$$

The measured power spectrum is

$$
\begin{aligned}
\left\langle\widetilde{\delta}_{n \lambda}^{\text {obs }} \widetilde{\delta}_{n^{\prime} \lambda}^{\text {obs }}\right\rangle= & \left\langle\widetilde{\delta}_{n \lambda} \widetilde{\delta}_{n^{\prime} \lambda}\right\rangle-A_{\lambda} \sum_{j} A_{\lambda j}^{-1} \sum_{i i^{\prime}} R_{n i}^{\lambda,-1} R_{n^{\prime} i^{\prime}}^{\lambda,-1}\left\langle\delta\left(\hat{\boldsymbol{r}}_{j}, z_{i}\right) \bar{\delta}\left(z_{i^{\prime}}\right)\right\rangle \\
& -A_{\lambda} \sum_{j} A_{\lambda j}^{-1} \sum_{i i^{\prime}} R_{n^{\prime} i}^{\lambda,-1} R_{n i^{\prime}}^{\lambda,-1}\left\langle\delta\left(\hat{\boldsymbol{r}}_{j}, z_{i}\right) \bar{\delta}\left(z_{i^{\prime}}\right)\right\rangle \\
& +A_{\lambda}^{2} \sum_{i i^{\prime}} R_{n i}^{\lambda,-1} R_{n^{\prime} i^{\prime}}^{\lambda,-1}\left\langle\bar{\delta}\left(z_{i}\right) \bar{\delta}\left(z_{i^{\prime}}\right)\right\rangle .
\end{aligned}
$$

With Eq. (4.34) the remaining angular transforms at $\lambda=0$ are of the form

$$
\sum_{j} A_{\lambda j}^{-1} \delta\left(\hat{\boldsymbol{r}}_{j}, z_{i}\right) \simeq \frac{\delta_{\lambda 0}^{K}}{\sqrt{4 \pi f_{\text {sky }}}} \int_{D} \mathrm{~d}^{2} \hat{r} \delta\left(\hat{\boldsymbol{r}}, z_{i}\right)=A_{\lambda} \bar{\delta}(z),
$$

where we used the definition of $\bar{\delta}(z)$ (Eq. (4.29)) for the last equality. Therefore, the last three terms in Eq. (4.36) can be combined, and we get

$$
\left\langle\widetilde{\delta}_{n \lambda}^{\mathrm{obs}} \widetilde{\delta}_{n^{\prime} \lambda}^{\mathrm{obs}}\right\rangle=\left\langle\widetilde{\delta}_{n \lambda} \widetilde{\delta}_{n^{\prime} \lambda}\right\rangle-A_{\lambda}^{2} \sum_{i i^{\prime}} R_{n i}^{\lambda,-1} R_{n^{\prime} i^{\prime}}^{\lambda,-1}\left\langle\bar{\delta}\left(z_{i}\right) \bar{\delta}\left(z_{i^{\prime}}\right)\right\rangle .
$$

Next, we express $\left\langle\bar{\delta}\left(z_{i}\right) \bar{\delta}\left(z_{i^{\prime}}\right)\right\rangle$ in terms of the power spectrum $C_{l n n^{\prime}}$. Discretizing Eq. (4.29) and expressing the configuration-space density contrast in terms of its cryotransform, we get

$$
\begin{aligned}
\left\langle\bar{\delta}\left(z_{i}\right) \bar{\delta}\left(z_{i^{\prime}}\right)\right\rangle & =\frac{\Omega_{\mathrm{pix}}^{2}}{\left(4 \pi f_{\mathrm{sky}}\right)^{2}} \sum_{j j^{\prime}}\left\langle\delta\left(\hat{\boldsymbol{r}}_{j}, z_{i}\right) \delta\left(\hat{\boldsymbol{r}}_{j^{\prime}}, z_{i^{\prime}}\right)\right\rangle \\
& =\frac{\Omega_{\mathrm{pix}}^{2}}{\left(4 \pi f_{\mathrm{sky}}\right)^{2}} \sum_{j j^{\prime}} \sum_{\Lambda \Lambda^{\prime}} \sum_{N N^{\prime}} A_{j \Lambda} A_{j^{\prime} \Lambda^{\prime}} R_{i N}^{\Lambda} R_{i^{\prime} N^{\prime}}^{\Lambda^{\prime}}\left\langle\widetilde{\delta}_{\Lambda N} \widetilde{\delta}_{\Lambda^{\prime} N^{\prime}}\right\rangle .
\end{aligned}
$$

Performing the sum over $j$ and $j^{\prime}$ first,

$$
\Omega_{\mathrm{pix}} \sum_{j} A_{j \Lambda}=\sqrt{4 \pi f_{\mathrm{sky}}} \delta_{\Lambda 0}^{K}=A_{\Lambda},
$$

we get

$$
\left\langle\bar{\delta}\left(z_{i}\right) \bar{\delta}\left(z_{i^{\prime}}\right)\right\rangle=\frac{1}{4 \pi f_{\mathrm{sky}}} \sum_{N N^{\prime}} R_{i N}^{0} R_{i^{\prime} N^{\prime}}^{0}\left\langle\widetilde{\delta}_{0 N} \widetilde{\delta}_{0 N^{\prime}}\right\rangle .
$$


That is, a smaller sky coverage leads to a larger variance of $\bar{\delta}$.

Finally, inserting into Eq. (4.38), we get

$$
\left\langle\widetilde{\delta}_{n \lambda}^{\text {obs }} \widetilde{\delta}_{n^{\prime} \lambda}^{\text {obs }}\right\rangle=\left(1-\delta_{\lambda 0}^{K}\right)\left\langle\widetilde{\delta}_{n \lambda} \widetilde{\delta}_{n^{\prime} \lambda}\right\rangle
$$

or

$$
C_{\lambda n n^{\prime}}^{\mathrm{obs}}=\left(1-\delta_{\lambda 0}^{K}\right) C_{\lambda n n^{\prime}}-\delta_{\lambda 0}^{K} N_{\lambda n n^{\prime}}^{\mathrm{shot}}
$$

where the shot noise is $N_{\lambda n n^{\prime}}^{\text {shot }}=\delta_{n n^{\prime}}^{K} / \bar{n}^{\text {true }}$ under our assumption of constant $\bar{n}^{\text {true }}$.

To summarize, to first order in cryospace the local average effect becomes very simple: the power in the $\ell=0$ modes vanishes, and we may get a negative number due to the subtraction of the shot noise.

\subsection{SFB Cryopower}

To test the $\operatorname{CryoFaB}$, we generate 5000 log-normal simulations [e.g., 8, 21, 22] in a cube with sidelength $3072 h^{-1} \mathrm{Mpc}$ and mesh size $N_{\text {mesh }}^{3}=512^{3}$ and number density $\bar{n}=10^{-3} h^{3} \mathrm{Mpc}^{-3}$. This allows for a radial selection function $r_{\min }=500 h^{-1} \mathrm{Mpc}$ and $r_{\max }=1500 h^{-1} \mathrm{Mpc}$. For our five example masks we choose the same radial top-hat selection function.

The cryonalysis is performed with 50 radial bins and angular resolution $n_{\text {side }}=32$. We bin the resulting power spectrum into bins with $\Delta \ell=1$ and $\Delta k=10^{-3} h \mathrm{Mpc}^{-1}$, and we restrict ourselves to $\ell \leq 1.5 n_{\text {side }}$.

We show the average over the 5000 simulations in Fig. 11. The $\ell=0$ modes are significantly affected by the local average effect, also called integral constraint. These modes are all $\sim-1 / \bar{n}$, in agreement with the results from Section 4.4 .

As for the 2D case on the sphere, the cryo-window is more than just a pixel window, and it additionally includes aspects of the survey geometry. However, the effect is not simply an additional suppression on large scales. It can also lead to an enhancement on very large scales.

For a better comparison between the estimator and model, in Fig. 12 we show the relative difference of each mode as a color in $\ell-k_{n \ell}$ space. We get at least percent-level agreement for $\ell \lesssim n_{\text {side }}$ and $k_{n \ell} \lesssim 0.08 \mathrm{~h} \mathrm{Mpc}^{-1}$. This is in agreement with the 2D case in Fig. 8, and we expect a larger range of useable modes when either increasing $n_{\text {side }}$ or the number of radial bins.

\subsection{Covariance matrix}

In this section we detail the derivation of the covariance matrix. Due to the typically complicated nature of the covariance matrix, we refer to the specific form here as the cryovariance matrix. We apologize in advance for the length and complexity of the mathematics involved. However, given the importance of the covariance matrix we feel it deserves a place in the main text.

Since the cryo-modes of a homogeneous field are uncoupled, that is, since

$$
\left\langle\widetilde{\delta}_{i} \widetilde{\delta}_{j}\right\rangle=\delta_{i j}^{K}\left\langle\widetilde{\delta}_{i}^{2}\right\rangle,
$$

the covariance between cryo-modes $i$ and $j$ is

$$
V_{i j}=\left\langle\widetilde{\delta}_{i}^{2} \widetilde{\delta}_{j}^{2}\right\rangle-\left\langle\widetilde{\delta}_{i}^{2}\right\rangle\left\langle\widetilde{\delta}_{j}^{2}\right\rangle=2 \widetilde{\delta}_{i j}^{K}\left\langle\widetilde{\delta}_{i}^{2}\right\rangle^{2}
$$



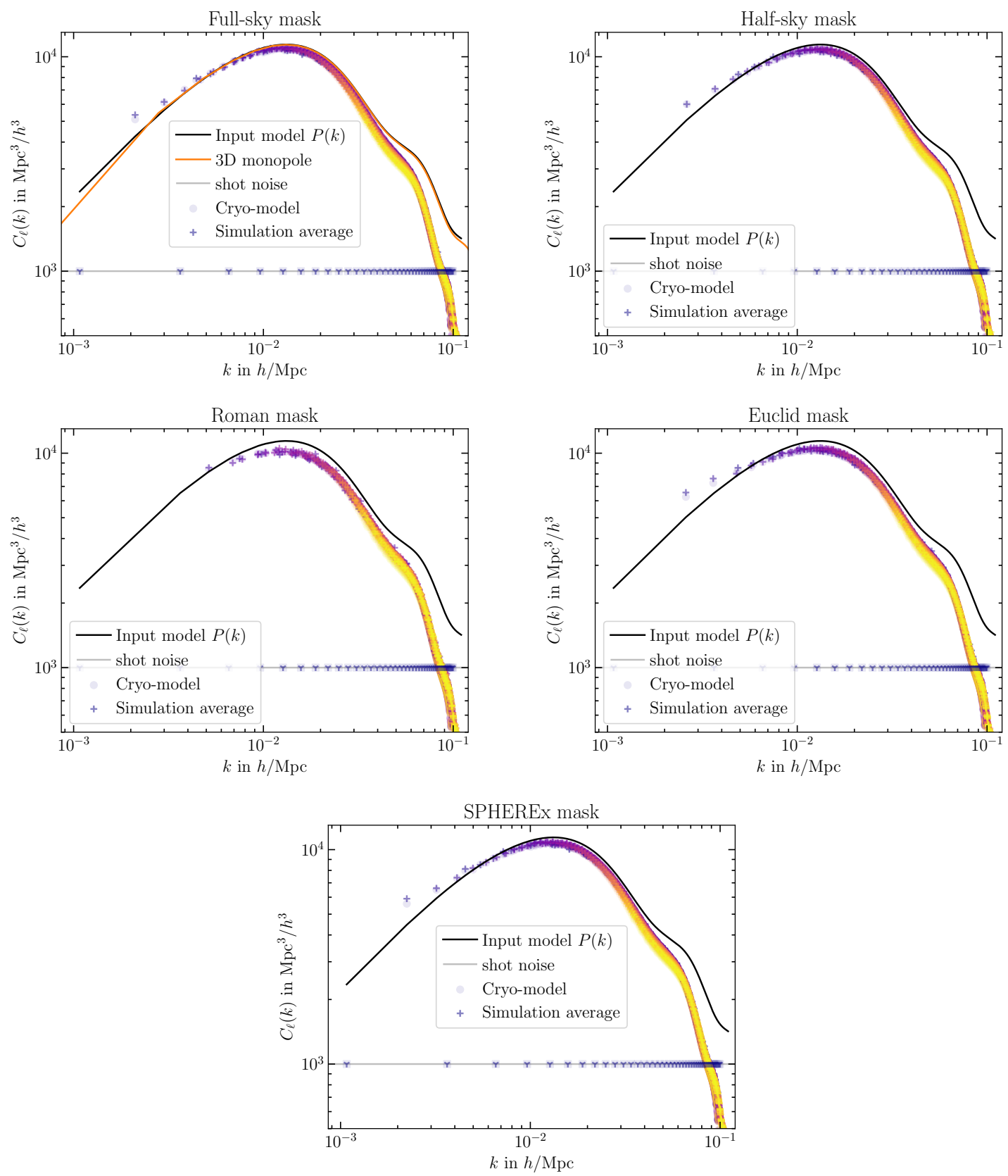

Figure 11. Here we show results for 5000 lognormal simulations for the five example masks. Each panel shows the input power spectrum, the shot noise, the average power spectrum measurement, and the model prediction. For the full-sky, we also show the 3D monopole measurement in order to verify our simulations. The modes have been binned into $\Delta \ell=1$ and $\Delta k=10^{-3} h^{-1} \mathrm{Mpc}$, putting the mark at the average $\ell$ and $k$ within each bin. The color indicates the $\ell$ mode, from $\ell=0$ (purple) to $\ell \sim 1.5 n_{\text {side }}$ (yellow). The measured modes and the model points are essentially on top of each other. The $\ell=0$ modes that are affected by the local average effect (or integral constraint) are negative due to the subtraction of the shot noise (shown as tri-down symbols for the estimator and squares for the model). To facilitate comparison between model and measurement for the rest of the modes, we refer to Fig. 12. 

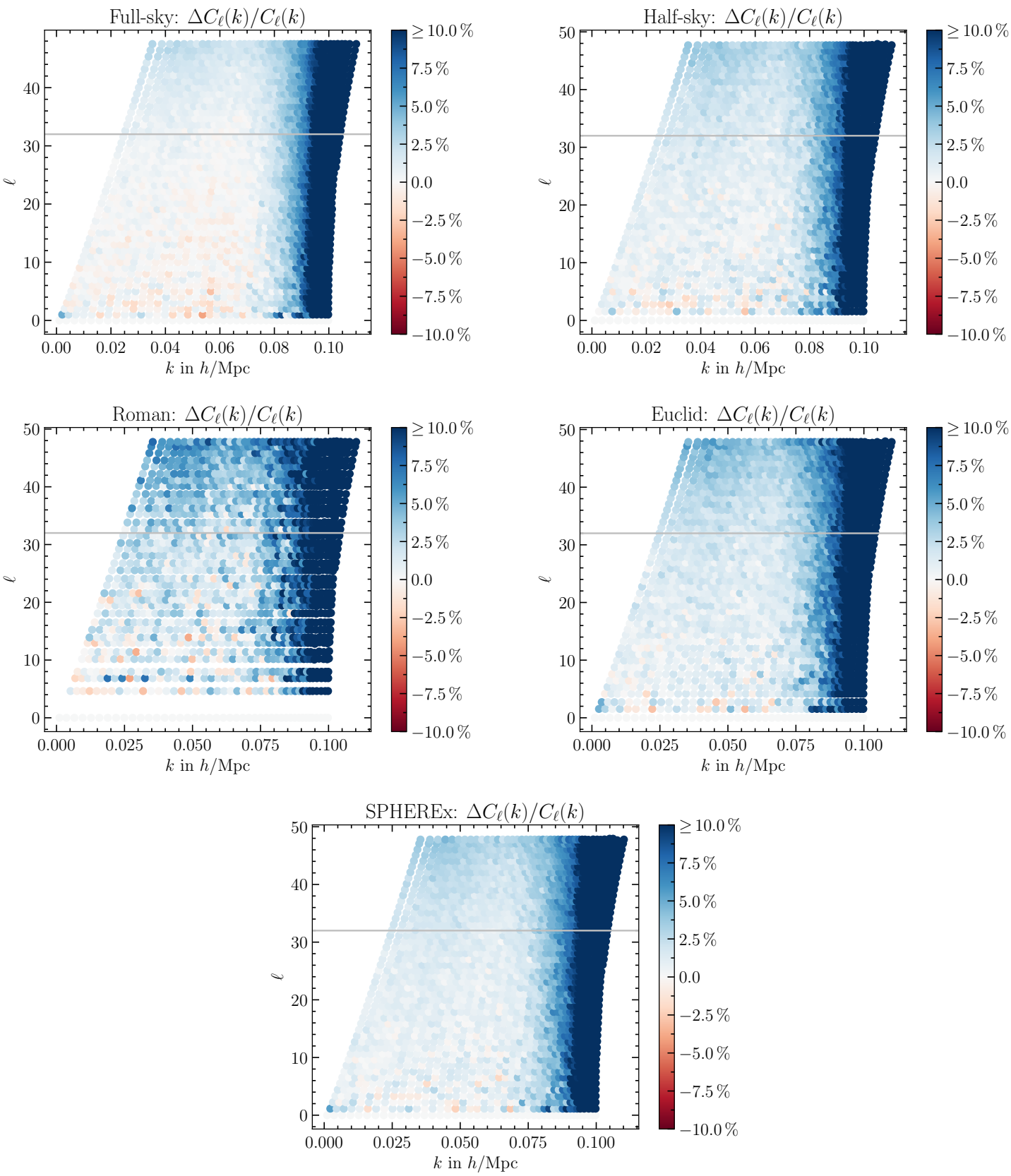

Figure 12. To assess the bias of the cryo-estimator, it is important to disentangle the $\ell-k$ dependence. Averaged over 5000 lognormal simulations for the five example masks, the color in each plot shows the relative error in the estimated mode. The modes are binned into $\Delta \ell=1$ and $\Delta k=10^{-3} h^{-1} \mathrm{Mpc}$. All plots show good agreement at low $\ell$ and low $k$. However, the noise is larger for smaller $\ell$, and the noise is especially large for the Roman-like mask, as it covers the smallest volume. The grey horizontal line indicates $n_{\text {side }}$, and all masks show a slight positive bias for $\ell>n_{\text {side }}$, similar to what was found for the 2D estimator in Fig. 8. Finally, all plots show a strong and rapidly growing bias at $k \gtrsim 0.09 \mathrm{~h} \mathrm{Mpc}^{-1}$, which we expect is due to the choice of radial resolution. Those modes will need to be discarded in a realistic application. 

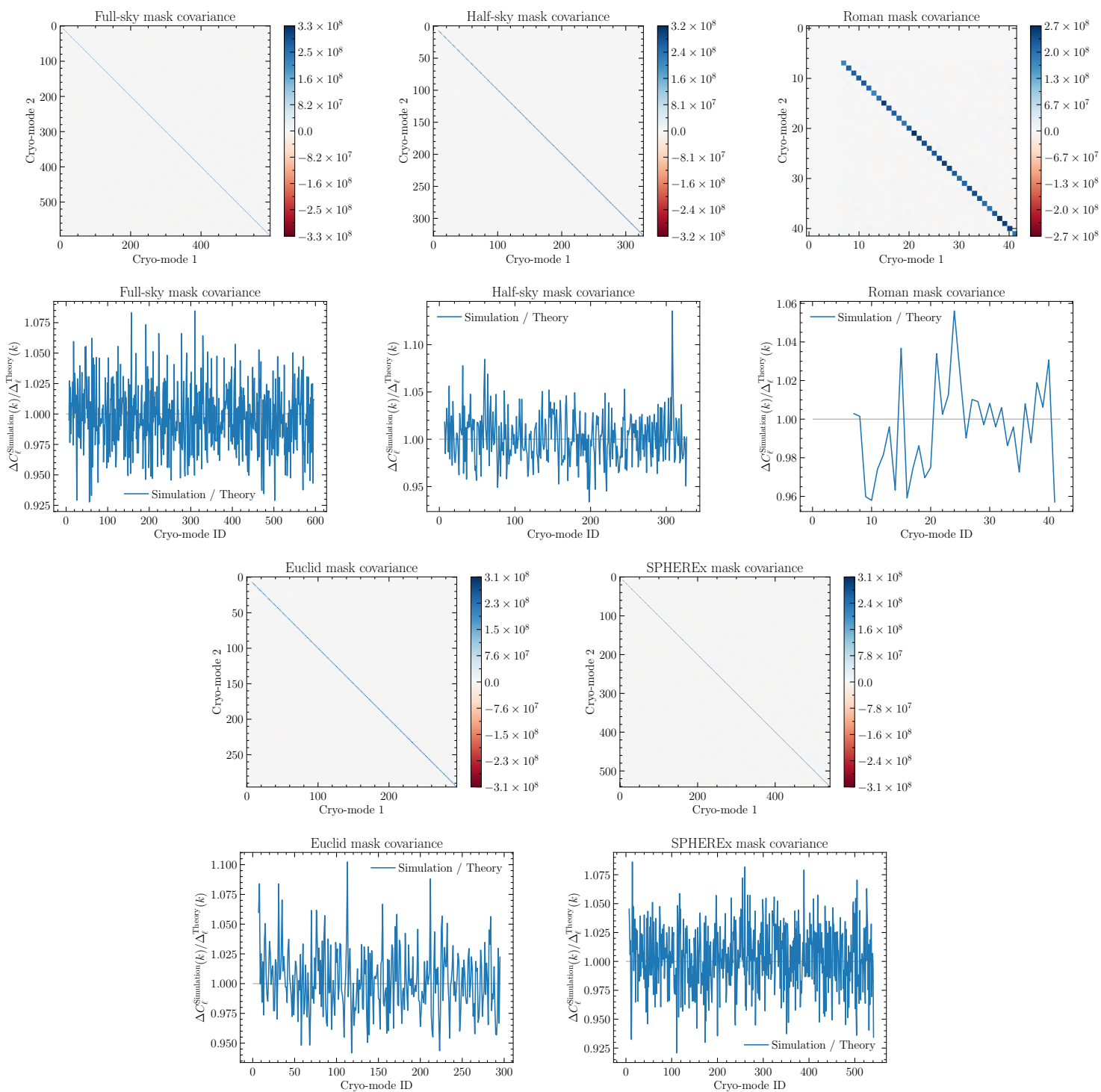

Figure 13. The cryovariance matrices for each of our five example surveys are shown in the first and third rows. Since the cryofunks eliminate the cross-correlations induced by the window function and the simulations do not contain a connected four-point part, all covariance matrices show the same structure: vanishing elements everywhere except on the diagonal. The second and fourth rows compare the measured diagonal with the predicted according to Eq. (4.46). The first few modes are $\ell=0$ and they are affected by the local average effect, or integral constraint. We have limited ourselves in these plots to the largest modes $\ell<10$ and $k<2 \times 10^{-2} h \mathrm{Mpc}^{-1}$. 
where we used Wick's theorem under the assumption of a Gaussian field, and $i$ and $j$ each stand for a tuple $\left(k_{n \ell}, \ell\right)$. That is, the covariance matrix is diagonal. Equivalently, the $1 \sigma$ variance is

$$
\sigma\left(C_{\ell}(k)\right) \sim \sqrt{\frac{2}{N_{k \ell}}}\left[C_{\ell}(k)+\frac{1}{\bar{n}}\right],
$$

where $N_{k \ell}$ is the number of modes collected into a bandpass in both $k$ and $\ell$.

We show a comparison with lognormal simulations in Fig. 13. In the figure, we show the full covariance matrices for each of our masks for the largest modes $\ell<10$ and $k<$ $2 \times 10^{-2} h \mathrm{Mpc}^{-1}$, as well as the ratio to the predicted value using Eq. (4.47) with $N_{i}=1$.

\section{Discussion}

Several points deserve some discussion. We discuss the nature of the boundary conditions, the interpretation of the angular momentum $\ell$ and the not-well-defined nature of the magnetic quantum number $m$, and the relation to the methods by [1].

\subsection{Non-Local Boundary Conditions}

The boundary conditions implied by our method are non-local [4] and not straightforward to interpret, as we show in the following. First, we define the Laplacian operator such that $\mathcal{L} f(\boldsymbol{r})=-\nabla_{\boldsymbol{r}}^{2} f(\boldsymbol{r})$, and we define the Green's operator $\mathcal{K}$ such that in $n$ dimensions

$$
\mathcal{K} f(\boldsymbol{r})=\int_{D} \mathrm{~d}^{n} r^{\prime} G\left(\boldsymbol{r}, \boldsymbol{r}^{\prime}\right) f\left(\boldsymbol{r}^{\prime}\right)
$$

Also, because $G$ is a function of $\boldsymbol{r}-\boldsymbol{r}^{\prime}$, we have $\nabla_{\boldsymbol{r}}^{2} G\left(\boldsymbol{r}, \boldsymbol{r}^{\prime}\right)=\nabla_{\boldsymbol{r}^{\prime}}^{2} G\left(\boldsymbol{r}, \boldsymbol{r}^{\prime}\right)$. Then, the commutator is

$$
\begin{aligned}
{[\mathcal{K} \mathcal{L}-\mathcal{L} \mathcal{K}] f(\boldsymbol{r}) } & =-\int_{D} \mathrm{~d}^{n} r^{\prime}\left[G\left(\boldsymbol{r}, \boldsymbol{r}^{\prime}\right) \nabla_{\boldsymbol{r}^{\prime}}^{2} f\left(\boldsymbol{r}^{\prime}\right)-\nabla_{\boldsymbol{r}^{\prime}}^{2} G\left(\boldsymbol{r}, \boldsymbol{r}^{\prime}\right) f\left(\boldsymbol{r}^{\prime}\right)\right] \\
& =-\int_{\partial D} \mathrm{~d}^{n-1} r^{\prime} \hat{\boldsymbol{n}} \cdot\left[G\left(\boldsymbol{r}, \boldsymbol{r}^{\prime}\right) \nabla_{\boldsymbol{r}^{\prime}} f\left(\boldsymbol{r}^{\prime}\right)-\nabla_{\boldsymbol{r}^{\prime}} G\left(\boldsymbol{r}, \boldsymbol{r}^{\prime}\right) f\left(\boldsymbol{r}^{\prime}\right)\right]
\end{aligned}
$$

where $\hat{\boldsymbol{n}}$ is the outward orthogonal unit vector to the boundary and we used Green's second identity Eq. (A.7). The left hand side must vanish for $\boldsymbol{r} \in D$ if the Laplacian and its Green's function are to commute, as they must if $f(\boldsymbol{r})$ is an eigenfunction. Then, for $\boldsymbol{r} \in \partial D$, we get the result that the boundary condition is

$$
\int_{\partial D} \mathrm{~d}^{n-1} r^{\prime} G\left(\boldsymbol{r}, \boldsymbol{r}^{\prime}\right) \hat{\boldsymbol{n}} \cdot \nabla_{\boldsymbol{r}^{\prime}} f\left(\boldsymbol{r}^{\prime}\right)=-\frac{1}{2} f(\boldsymbol{r})+\int_{\partial D} \mathrm{~d}^{n-1} r^{\prime} \hat{\boldsymbol{n}} \cdot \nabla_{\boldsymbol{r}^{\prime}} G\left(\boldsymbol{r}, \boldsymbol{r}^{\prime}\right) f\left(\boldsymbol{r}^{\prime}\right) .
$$

The extra term $-\frac{1}{2} f(\boldsymbol{r})$ appears when moving $\boldsymbol{r}$ from the interior of $D$ to the boundary. We refer the reader to [4, App. A] and references therein for a detailed derivation.

A basis function must both be an eigenfunction to the Laplacian and satisfy the nonlocal boundary condition Eq. (5.4). Evidently, Green's function $G\left(\boldsymbol{r}, \boldsymbol{r}^{\prime}\right)$ plays a crucial role in defining the boundary condition. Indeed, any harmonic function, that is, one that satisfies $\nabla^{2} h=0$, can be added to the Green's function (thus also modifying the Laplacian on the boundary), and that would lead to a different boundary condition. [4] lists some examples, as is our procedure in Section 3.3 to obtain the monopole. 


\subsection{Interpretation of effective $\ell$ and $m$}

Here we argue in a qualitative manner that the magnetic quantum number $m$ is not welldefined, and that the identification of our effective $\ell$ in Eq. (3.25) is justified as a measure of angular scale.

Unlike the standard spherical harmonics $Y_{\ell m}(\hat{\boldsymbol{r}})$, our eigenfunctions to the Laplacian are not eigenfunctions to the $z$-component of the angular momentum operator, and, therefore, the eigenfunctions do not have well-defined $m$. Instead, the $m$ label potentially degenerate modes $\ell$. Of course, for a power spectrum analysis of an isotropic field this does not pose a problem, because the result does not depend on the magnetic quantum number $m$. When moving to $3 \mathrm{D}$, the $\ell$ modes would in general be similarly indeterminate. This poses a problem for the SFB power spectrum, as it does depend on both $k$ and $\ell$. Therefore, we have opted to restrict ourselves to separable radial and angular selections.

The Laplacian eigenfunctions satisfy $\nabla^{2} f=-\ell(\ell+1) f$ on the full and partial spheres. Indeed, this equation is satisfied everywhere inside the survey. Hence, the $\ell$ must probe similar scales, whether it is the full sky or the partial sky. We, therefore, argue that the identification Eq. (3.25) leads to $\ell$ that probe comparable angular scales. Visual inspection of the eigenfunctions Figs. 1-5 agrees with this interpretation.

A related question is the relation to the associated Legendre polynomials of the first and second kinds, $P_{\ell}^{m}(\cos \theta)$ and $Q_{\ell}^{m}(\cos \theta)$, for non-integer $\ell$. Taking just the angular part of the Laplacian Eq. (4.1) and changing variables to $\mu=\cos \theta$, we get

$$
0=\left(1-\mu^{2}\right) \frac{\partial^{2} f}{\partial \mu^{2}}-2 \mu \frac{\partial f}{\partial \mu}+\lambda f+\frac{1}{1-\mu^{2}} \frac{\partial^{2} f}{\partial \phi^{2}},
$$

for an eigenfunction $f(\mu, \phi)$ of the Laplacian, $\nabla^{2} f=-\lambda f$. In general, our eigenfunctions are not eigenfunctions of the $\hat{\boldsymbol{z}}$-component of the angular momentum operator. Therefore, if we write $\partial^{2} f / \partial \phi^{2}=-m^{2} f$, then, in general, we expect $m^{2}$ to be a function of $\phi$. The question is, whether $m^{2}$ is a function of $\mu$. If it is not, then the identification $\lambda=\ell(\ell+1)$ is exact for non-integer $\ell$, because

$$
0=\left(1-\mu^{2}\right) \frac{\partial^{2} f}{\partial \mu^{2}}-2 \mu \frac{\partial f}{\partial \mu}+\ell(\ell+1) f-\frac{m^{2}}{1-\mu^{2}} f
$$

is the associated Legendre equation as long as $m^{2}$ is independent of $\mu=\cos \theta$. We conjecture that $m^{2}$ is indeed independent of $\mu$.

\subsection{Relation to [1]}

A similar goal was pursued in [1], with a different approach to obtaining an orthonormal basis supported on the domain of the survey. They start with the coupling matrix

$$
\mathbf{C}=\int_{D} \mathrm{~d}^{2} \hat{r} \mathbf{y}(\hat{\boldsymbol{r}}) \mathbf{y}^{T}(\hat{\boldsymbol{r}}),
$$

where the integration is over the domain of the survey, and the observed harmonic coefficients are $\mathbf{k}^{\text {obs }}=\mathbf{C k}$ for a full-sky analysis on the partial sky. Then, they find linear combinations of the spherical harmonics $\mathbf{y}^{\prime}(\hat{\boldsymbol{r}})=\mathbf{B y}(\hat{\boldsymbol{r}})^{6}$ such that the coupling matrix in this new basis

\footnotetext{
${ }^{6}$ This is a different $\mathbf{B}$ than in Eq. (3.18).
} 
becomes the identity,

$$
\widetilde{\mathbf{C}}=\int_{D} \mathrm{~d}^{2} \hat{r} \mathbf{y}^{\prime}(\hat{\boldsymbol{r}}) \mathbf{y}^{\prime T}(\hat{\boldsymbol{r}})=\mathbf{B C B}^{T}=\mathbf{I}
$$

The goal then is to find an $N_{\text {pix }}^{\text {survey }} \times N_{\text {pix }}^{\text {full-sky }}$ matrix B that satisfies Eq. (5.8). Their various approaches use the eigendecomposition of $\mathbf{C}$ or some approximation thereof.

In this regard, their approach and ours agree, since our basis functions are orthonormal over $D$ by design and Eq. (5.8) is satisfied. In this sense, our method is a special case of the more general approach presented in [1].

The specific form of our $\mathbf{B}$ that contains the coefficients of the linear combination of spherical harmonics is obtained as follows. We expand the Green's function $G\left(\hat{\boldsymbol{r}}, \hat{\boldsymbol{r}}^{\prime}\right)$ of the operator $\nabla^{2}$ in terms of spherical harmonics $Y_{\ell m}(\hat{\boldsymbol{r}})$,

$$
G\left(\hat{\boldsymbol{r}}, \hat{\boldsymbol{r}}^{\prime}\right)=\sum_{\ell m} Y_{\ell m}(\hat{\boldsymbol{r}}) a_{\ell m}\left(\hat{\boldsymbol{r}}^{\prime}\right)
$$

for some coefficient functions $a_{\ell m}(\hat{\boldsymbol{r}})$. Apply $\nabla^{2}$,

$$
\nabla_{\hat{\boldsymbol{r}}}^{2} G\left(\hat{\boldsymbol{r}}, \hat{\boldsymbol{r}}^{\prime}\right)=\sum_{\ell m} \nabla_{\hat{\boldsymbol{r}}}^{2} Y_{\ell m}(\hat{\boldsymbol{r}}) a_{\ell m}\left(\hat{\boldsymbol{r}}^{\prime}\right)=\sum_{\ell m} \ell(\ell+1) Y_{\ell m}(\hat{\boldsymbol{r}}) a_{\ell m}\left(\hat{\boldsymbol{r}}^{\prime}\right)
$$

By definition of the Green's function Eq. (3.1), and the orthogonality of the eigenfunctions, we have

$$
\nabla_{\hat{\boldsymbol{r}}}^{2} G\left(\hat{\boldsymbol{r}}, \hat{\boldsymbol{r}}^{\prime}\right)=-\delta^{D}\left(\hat{\boldsymbol{r}}^{\prime}-\hat{\boldsymbol{r}}\right)=-\sum_{\ell m} Y_{\ell m}(\hat{\boldsymbol{r}}) Y_{\ell m}^{*}\left(\hat{\boldsymbol{r}}^{\prime}\right)
$$

Setting Eqs. (5.10) and (5.11) equal, multiplying by $Y_{L M}^{*}(\hat{\boldsymbol{r}})$, and integrating over $\hat{\boldsymbol{r}}$, we get

$$
L(L+1) a_{L M}\left(\hat{\boldsymbol{r}}^{\prime}\right)=-Y_{L M}^{*}\left(\hat{\boldsymbol{r}}^{\prime}\right) .
$$

Therefore, Eq. (5.9) becomes

$$
G\left(\hat{\boldsymbol{r}}, \hat{\boldsymbol{r}}^{\prime}\right)=-\sum_{\ell m} \frac{Y_{\ell m}(\hat{\boldsymbol{r}}) Y_{\ell m}^{*}\left(\hat{\boldsymbol{r}}^{\prime}\right)}{\ell(\ell+1)}
$$

Inserting Eq. (5.13) into Eq. (3.4),

$$
\begin{aligned}
Z_{\lambda}(\hat{\boldsymbol{r}}) & =-\lambda \int_{D} \mathrm{~d}^{2} \hat{\boldsymbol{r}}^{\prime} \sum_{\ell m} \frac{Y_{\ell m}(\hat{\boldsymbol{r}}) Y_{\ell m}^{*}\left(\hat{\boldsymbol{r}}^{\prime}\right)}{\ell(\ell+1)} Z_{\lambda}\left(\hat{\boldsymbol{r}}^{\prime}\right) \\
& =\sum_{\ell m}\left[\frac{-\lambda}{\ell(\ell+1)} \int_{D} \mathrm{~d}^{2} \hat{\boldsymbol{r}}^{\prime} Y_{\ell m}^{*}\left(\hat{\boldsymbol{r}}^{\prime}\right) Z_{\lambda}\left(\hat{\boldsymbol{r}}^{\prime}\right)\right] Y_{\ell m}(\hat{\boldsymbol{r}}),
\end{aligned}
$$

where we relabeled the cryofunction as $Z_{\lambda}(\hat{\boldsymbol{r}})$ to avoid confusion with the spherical harmonics $Y_{\ell m}(\hat{\boldsymbol{r}})$. The term in square brackets is some constant coefficient $c_{\lambda, \ell m}$ that expresses the cryofunctions in terms of spherical harmonics. 


\section{Conclusion}

In this paper we have developed a proof-of-concept of using eigenfunctions of the Laplacian adapted to the exact geometry of a survey. That is, we fit the Fourier-transform box exactly onto the survey geometry. On the sphere, we obtain linear combinations of the real spherical harmonics with effectively non-integer $\ell$. We show some of the eigenfunctions in Figs. 1-5.

In this limit both the 2-point function in harmonic space and its covariance matrix become diagonal for a Gaussian random field (see Figs. 7 and 13). Poissonian shot noise also takes on the simple form $1 / \bar{n}$, and the first-order local average effect can be treated exactly analytically. This comes at the expense of a somewhat complex pixel and window function, which is not straightforward to invert. However, the simplicity of the covariance matrix gives hope for a relatively efficient generalization to higher order statistics, though we have not looked further into this.

Our approach builds on the work by $[4,5]$ in the applied mathematics and climate science literature. Compared to them we formalize the symmetry of the discretized Green's function $G_{i j}$, generalize to HEALPix, and we develop the pixel/geometry window and covariance matrix.

We also develop a 3D SFB power spectrum estimator by using the approach separately in the radial and angular directions. We get essentially unbiased results for $\ell \lesssim n_{\text {side, }}$, as shown in Figs. 11 and 12. However, we have assumed a perfectly homogeneous universe, and this will need to be generalized if applied to a real survey with, e.g., growth of structure along the light cone.

The cryo-approach is dependent on calculating eigenfunctions of the Green's matrix of size $N_{\text {pix }} \times N_{\text {pix }}$, where $N_{\text {pix }}$ is the number of pixels in the survey. Therefore, only the largest scales are computationally feasible: a full-sky survey at resolution $n_{\text {side }}=32$ will require $1.2 \mathrm{~GB}$ of storage; at the next higher resolution this becomes $19 \mathrm{~GB}$; at $n_{\text {side }}=128$ it is 309 GB using 64-bit floats for the angular transform. This is a limitation of our current implementation on a modern laptop, and it can likely be optimized. In any case, these need to be computed only once for a given mask, and can therefore be precomputed.

We have assumed a binary mask and selection function throughout. However it is straightforward to generalize the method to non-binary masks, e.g., when stars block out part of a pixel. We leave this to a future paper.

Our code will be available publically at https://github.com/hsgg/CryoFaBs.jl, once approved for release by our institution.

\section{Acknowledgments}

(C)2021. All rights reserved. Part of this work was done at Jet Propulsion Laboratory, California Institute of Technology, under a contract with the National Aeronautics and Space Administration. This work was supported by NASA grant 15-WFIRST15-0008 Cosmology with the High Latitude Survey Roman Science Investigation Team (SIT). Henry S. G. Gebhardt's research was supported by an appointment to the NASA Postdoctoral Program at the Jet Propulsion Laboratory, administered by Universities Space Research Association under contract with NASA. 


\section{References}

[1] D.J. Mortlock, A.D. Challinor and M.P. Hobson, Analysis of cosmic microwave background data on an incomplete sky, MNRAS 330 (2002) 405 [astro-ph/0008083].

[2] H.S. Grasshorn Gebhardt and O. Doré, SuperFaB: a fabulous code for Spherical Fourier-Bessel decomposition, arXiv e-prints (2021) arXiv:2102.10079 [2102.10079].

[3] L. Samushia, Proper Fourier decomposition formalism for cosmological fields in spherical shells, arXiv e-prints (2019) arXiv:1906.05866 [1906.05866].

[4] N. Saito, Data analysis and representation on a general domain using eigenfunctions of laplacian, Applied and Computational Harmonic Analysis 25 (2008) 68.

[5] T. DelSole and M.K. Tippett, Laplacian Eigenfunctions for Climate Analysis, Journal of Climate 28 (2015) 7420.

[6] K.M. Górski, E. Hivon, A.J. Banday, B.D. Wandelt, F.K. Hansen, M. Reinecke et al., HEALPix: A Framework for High-Resolution Discretization and Fast Analysis of Data Distributed on the Sphere, ApJ 622 (2005) 759 [astro-ph/0409513].

[7] H.S. Grasshorn Gebhardt and D. Jeong, Nonlinear redshift-space distortions in the harmonic-space galaxy power spectrum, Phys. Rev. D 102 (2020) 083521 [2008.08706].

[8] A. Agrawal, R. Makiya, C.-T. Chiang, D. Jeong, S. Saito and E. Komatsu, Generating log-normal mock catalog of galaxies in redshift space, J. Cosmology Astropart. Phys. 2017 (2017) 003 [1706.09195].

[9] P.J.E. Peebles, Statistical Analysis of Catalogs of Extragalactic Objects. I. Theory, ApJ 185 (1973) 413.

[10] H.A. Feldman, N. Kaiser and J.A. Peacock, Power-Spectrum Analysis of Three-dimensional Redshift Surveys, ApJ 426 (1994) 23 [astro-ph/9304022].

[11] K. Yamamoto, M. Nakamichi, A. Kamino, B.A. Bassett and H. Nishioka, A Measurement of the Quadrupole Power Spectrum in the Clustering of the 2dF QSO Survey, PASJ 58 (2006) 93 [astro-ph/0505115].

[12] E. Castorina and M. White, Beyond the plane-parallel approximation for redshift surveys, MNRAS 476 (2018) 4403 [1709.09730].

[13] F. Beutler, E. Castorina and P. Zhang, Interpreting measurements of the anisotropic galaxy power spectrum, J. Cosmology Astropart. Phys. 2019 (2019) 040 [1810.05051].

[14] K.B. Fisher, O. Lahav, Y. Hoffman, D. Lynden-Bell and S. Zaroubi, Wiener reconstruction of density, velocity and potential fields from all-sky galaxy redshift surveys, MNRAS 272 (1995) 885 [astro-ph/9406009].

[15] F. Beutler, S. Saito, H.-J. Seo, J. Brinkmann, K.S. Dawson, D.J. Eisenstein et al., The clustering of galaxies in the SDSS-III Baryon Oscillation Spectroscopic Survey: testing gravity with redshift space distortions using the power spectrum multipoles, MNRAS 443 (2014) 1065 [1312.4611].

[16] A. de Mattia and V. Ruhlmann-Kleider, Integral constraints in spectroscopic surveys, J. Cosmology Astropart. Phys. 2019 (2019) 036 [1904.08851].

[17] R. de Putter, C. Wagner, O. Mena, L. Verde and W.J. Percival, Thinking outside the box: effects of modes larger than the survey on matter power spectrum covariance, J. Cosmology Astropart. Phys. 2012 (2012) 019 [1111.6596].

[18] D. Wadekar, M.M. Ivanov and R. Scoccimarro, Cosmological constraints from BOSS with analytic covariance matrices, Phys. Rev. D 102 (2020) 123521 [2009.00622]. 
[19] V. Desjacques, Y.B. Ginat and R. Reischke, Statistics of a single sky: constrained random fields and the imprint of Bardeen potentials on galaxy clustering, MNRAS 504 (2021) 5612 [2009.02036].

[20] A. Taruya, T. Nishimichi and D. Jeong, Covariance of the matter power spectrum including the survey window function effect: $N$-body simulations versus fifth-order perturbation theory on grids, Phys. Rev. D 103 (2021) 023501 [2007.05504].

[21] P. Coles and B. Jones, A lognormal model for the cosmological mass distribution., MNRAS 248 (1991) 1.

[22] H.S. Xavier, F.B. Abdalla and B. Joachimi, Improving lognormal models for cosmological fields, MNRAS 459 (2016) 3693 [1602.08503].

[23] "NIST Digital Library of Mathematical Functions." http://dlmf.nist.gov/, Release 1.1.1 of 2021-03-15.

\section{A Useful formulae}

Spherical Bessel functions and spherical harmonics satisfy orthogonality relations

$$
\begin{aligned}
\delta^{D}\left(k-k^{\prime}\right) & =\frac{2 k k^{\prime}}{\pi} \int_{0}^{\infty} \mathrm{d} r r^{2} j_{\ell}(k r) j_{\ell}\left(k^{\prime} r\right) \\
\delta_{\ell \ell^{\prime}}^{K} \delta_{m m^{\prime}}^{K} & =\int \mathrm{d} \Omega_{\hat{\boldsymbol{r}}} Y_{\ell m}(\hat{\boldsymbol{r}}) Y_{\ell^{\prime} m^{\prime}}^{*}(\hat{\boldsymbol{r}}) .
\end{aligned}
$$

Real spherical harmonics are defined as

$$
Y_{\ell m}^{\text {real }}(\theta, \phi)= \begin{cases}\sqrt{2}(-1)^{m} \operatorname{Im}\left[Y_{\ell|m|}(\theta, \phi)\right] & \text { if } m<0, \\ Y_{\ell 0}(\theta, \phi) & \text { if } m=0, \\ \sqrt{2}(-1)^{m} \operatorname{Re}\left[Y_{\ell m}(\theta, \phi)\right] & \text { if } m>0 .\end{cases}
$$

An alternative to the Haversine formula Eq. (3.12) is

$$
\rho=\arctan \frac{x}{y}
$$

where

$$
\begin{aligned}
& x=\left(\left[\sin \theta^{\prime} \sin \Delta \phi\right]^{2}+\left[\sin \theta \cos \theta^{\prime}-\cos \theta \sin \theta^{\prime} \cos \Delta \phi\right]^{2}\right)^{\frac{1}{2}}, \\
& y=\cos \theta \cos \theta^{\prime}+\sin \theta \sin \theta^{\prime} \cos \Delta \phi
\end{aligned}
$$

This requires proper inversion of the tangent, which in Julia is implemented as atan $(\mathrm{x}, \mathrm{y})$.

Green's second identity in three dimensions is

$$
\int_{D} \mathrm{~d}^{3} r\left(\psi \nabla^{2} \varphi-\varphi \nabla^{2} \psi\right)=\oint_{\partial D} \mathrm{~d}^{2} \hat{r} \hat{\boldsymbol{n}} \cdot(\psi \nabla \varphi-\varphi \nabla \psi),
$$

where $\hat{\boldsymbol{n}}$ is the outward-directed unit vector on the boundary at $\boldsymbol{r}$.

\section{B Radial Green's function}

We derive the radial Green's function using two different techniques. 


\section{B.1 First derivation}

Express the Green's function in terms of its spherical Bessel transform,

$$
G\left(r, r^{\prime}\right)=\int \mathrm{d} k j_{\ell}(k r) \widetilde{G}\left(k, r^{\prime}\right) .
$$

Inserting into the defining equation for the Green's function,

$$
\int \mathrm{d} k \widetilde{G}\left(k, r^{\prime}\right)\left[-k^{2} j_{\ell}(k r)\right]=-\delta^{D}\left(r^{\prime}-r\right) .
$$

Integrate over $\frac{2 k^{\prime 2}}{\pi} \int \mathrm{d} r r^{2} j_{\ell}\left(k^{\prime} r\right)$ to get

$$
\widetilde{G}\left(k^{\prime}, r^{\prime}\right)=\frac{2 r^{\prime 2}}{\pi} j_{\ell}\left(k^{\prime} r^{\prime}\right)
$$

and

$$
G\left(r, r^{\prime}\right)=\frac{2 r^{\prime 2}}{\pi} \int_{0}^{\infty} \mathrm{d} k^{\prime} j_{\ell}\left(k^{\prime} r\right) j_{\ell}\left(k^{\prime} r^{\prime}\right)=\frac{r^{\prime 2}}{r} \int_{0}^{\infty} \mathrm{d} k^{\prime} k^{\prime-1} J_{\nu}\left(k^{\prime} r\right) J_{\nu}\left(k^{\prime} r^{\prime}\right),
$$

where $\nu=\ell+\frac{1}{2}$, and we used that the spherical Bessel function is related to the cylindrical Bessel function by $j_{\ell}(k r)=\sqrt{\frac{\pi}{2 k r}} J_{\ell+\frac{1}{2}}(k r)$. The integral has an analytic solution for $r \leq r^{\prime}$ [23, Eqs. 10.22.56 and 10.22.57]

$$
\frac{1}{2 \nu}\left(\frac{r}{r^{\prime}}\right)^{\nu}
$$

Furthermore, the integral is symmetric under exchange of $r$ and $r^{\prime}$. Therefore, Eq. (B.4) becomes

$$
G\left(r, r^{\prime}\right)= \begin{cases}\frac{r^{\prime}}{2 \ell+1}\left(\frac{r}{r^{\prime}}\right)^{\ell} & \text { for } r \leq r^{\prime}, \\ \frac{r^{\prime}}{2 \ell+1}\left(\frac{r}{r^{\prime}}\right)^{-\ell-1} & \text { for } r^{\prime} \leq r .\end{cases}
$$

\section{B.2 Another derivation}

The solution is the solution to the homogeneous differential equation in the two regimes $r<r^{\prime}$ and $r>r^{\prime}$,

$$
G\left(r, r^{\prime}\right)= \begin{cases}A r^{\ell}+B r^{-\ell-1}, & \text { if } 0 \leq r<r^{\prime}, \\ C r^{\ell}+D r^{-\ell-1}, & \text { if } r>r^{\prime},\end{cases}
$$

for some $A, B, C$, and $D$. Requiring finite Green's function at $r=0$ and $r \rightarrow \infty$,

$$
B=C=0,
$$

when $\ell \neq 0$. Continuity at $r=r^{\prime}$ demands

$$
A r^{\ell \ell}=D r^{\prime-\ell-1} \text {. }
$$

Next, integrate Eq. (4.4) over a small interval around $r^{\prime}$, more precisely the interval $\lim _{\epsilon \rightarrow 0}\left[r^{\prime}-\right.$ $\left.\epsilon, r^{\prime}+\epsilon\right]$ for $\epsilon>0$. We find

$$
\lim _{\epsilon \rightarrow 0^{+}}\left[r^{2} \frac{\mathrm{d}}{\mathrm{d} r} G\left(r, r^{\prime}\right)\right]_{r=r^{\prime}-\epsilon}^{r=r^{\prime}+\epsilon}=-r^{\prime 2}
$$


or more explicitly,

$$
A \ell r^{\ell \ell-1}+D(\ell+1) r^{\prime-\ell-2}=1 .
$$

for $\ell \geq 1$. Eqs. (B.9) and (B.11) are solved by

$$
\begin{aligned}
A & =\frac{r^{\prime}}{2 \ell+1} r^{\prime-\ell}, \\
D & =\frac{r^{\prime}}{2 \ell+1} r^{\ell \ell+1} .
\end{aligned}
$$

Therefore,

$$
G\left(r, r^{\prime}\right)= \begin{cases}\frac{r^{\prime}}{2 \ell+1}\left(\frac{r}{r^{\prime}}\right)^{\ell} & \text { for } r \leq r^{\prime} \\ \frac{r^{\prime}}{2 \ell+1}\left(\frac{r}{r^{\prime}}\right)^{-\ell-1} & \text { for } r^{\prime} \leq r\end{cases}
$$

